Carsten Peust

\title{
Direkter und indirekter Genitiv im Älteren Ägyptisch nach dem Zeugnis der Sargtexte
}

https://doi.org/10.1515/zaes-2017-0013

Summary: Based on the Coffin Text corpus, the use of the two genitive constructions (direct vs. indirect) of Earlier Egyptian is investigated. It is argued that there is no functional difference whatsoever between them, but rather that the lexeme employed in the first position is the decisive factor. Two classes of nouns are identified, one of them usually selecting the direct genitive and the other the indirect genitive. Irrespectively of these classes, the indirect genitive is always required when the nomen regens is complex. On the other hand, the complexity of the nomen rectum does not influence the choice of the genitive construction. Contrary to these rules, the indirect genitive is sometimes preferred in order to prevent ambiguity that might otherwise have occurred.

Keywords: Egyptian - Grammar - Genitive

Das Ältere Ägyptisch besitzt bekanntlich zwei Genitivkonstruktionen: (1) den direkten Genitiv, bei dem das nomen regens (im Folgenden auch: Erstnomen) und das nomen rectum (= der Genitiv, auch: Zweitnomen) unmittelbar hintereinandergestellt werden, und (2) den indirekten Genitiv, bei dem zwischen beide eine mit dem Erstnomen kongruierende nota genitivi $n$ (fem. n.t, masc. pl. n.w) tritt $^{1}$.

Über den Funktionsunterschied zwischen beiden Konstruktionen herrscht beträchtliche Unklarheit. Gunn $(1949,24)$ bezeichnet die Frage nach der Wahl der Genitivkonstruktion als „one of the most important outstanding problems of Egyptian grammar“. Edel (1955/64, §318, mit fast gleichem Wortlaut auch Barta 1995, 29) stellt fest: „Leider lässt sich keine allgemein gültige Regel aufstellen,

1 Die Kongruenz mag oft überflüssig sein und wurde im weiteren Verlauf der ägyptischen Sprachgeschichte auch aufgegeben, kann aber manchmal die Konstituentenstruktur klären wie in w3.wt hrj.t $m w$ n.t $R$-st3.w (CT VII 348e, VII 521f), was also heißt „die [Wege auf dem Wasser] von Ra-Setjau“ und nicht „die Wege auf dem [Wasser von Ra-Setjau]“, oder hrj.t k3.w n.t ntr.w (CT VI 15c) „die [Vorsteherin der Speisen] der Götter“ und nicht „die Vorsteherin der [Speisen der Götter]“.

Carsten Peust, E-Mail: cpeust@gmx.de wann der eine oder der andere Genetiv steht“, und bei Callender (1975, 114 Anm. 18) heißt es: „A full investigation of Egyptian genitival constructions is sorely needed.“ Diese Einschätzung hat sich bis in die neueste Zeit wenig geändert: „Unfortunately, the distribution of the two patterns is far from being understood.“ (Egedi 2010, 6); „Wann Genetivverbindung und wann Genetivadjektiv [= indirekter Genitiv, C.P.] verwendet wird, ist nicht «voraussehbar» [...] für die Wahl der einen oder anderen Konstruktion dürften jeweils Gründe des Sprachrhythmus oder der Lautkombination verantwortlich sein, die dem individuellen Sprachempfinden des Individuums zuzugestehen sind.“ (Jenni 2010, 73). Es ist auch darauf hingewiesen worden, dass beide Genitivkonstruktionen anscheinend als freie Varianten auftreten können, also bei gleichem Regens und gleichem Rectum (Beispiele etwa bei Westendorf 1962, 93), wenn auch vielleicht nicht wirklich innerhalb vollkommen synchroner Korpora.

Immer wieder ist der Eindruck erweckt worden, der direkte Genitiv sei mehr oder weniger marginal oder auf feste Verbindungen beschränkt: „Der alte Genetivausdruck, bei dem das regierte Nomen unverknüpft auf das regierende folgt, ist im Gebrauche beschränkt. Er findet sich augenscheinlich besonders in häufigen Verbindungen“ (Erman 1889, 59); „The direct genitive [...] is usual wherever the connexion between governing and governed noun is particularly close, as in titles, set phrases, etc." (Gardiner 1957, §85); „le génitif indirect est, dès l'époque ancienne, d'un usage beaucoup plus courant que le génitif direct“ (Lefebvre 1955, §146); „Two types of genitive constructions are attested for Middle Egyptian. The first sort consists of the juxtaposition of two noun phrases and seems to be marginally productive at best; many of these juxtapositions seem best considered as compounds." (Callender 1975, 114); „The construct state may once, long before writing, have been the rule. But already in Old and Middle Egyptian, the analytic type $p r n j t=j$ «my father's house» is dominant.“ (Depuydt 1999, 280); „Der direkte Genitiv steht vor allem in mehr oder minder festen oder nach bestimmten Produktionsmustern gewonnenen Ausdrücken [...] darüber hinaus ist er nur in engem Rahmen noch produktiv“ (Schenkel 2012, 132).

Grundsätzlich könnte man sich vorstellen, dass beide Genitivkonstruktionen verschiedene Arten des Genitivs 
bezeichnen würden, also irgendein funktionaler Unterschied bestünde. Ich nenne dies die funktionale Hypothese. Eine solche ist in der Tat verschiedentlich postuliert worden. Eine global weit verbreitete und daher a priori naheliegende Unterscheidung, zumindest aus einer modernen linguistischen Perspektive, wäre die zwischen alienabler Possession (von erworbenem Besitz) und inalienabler Possession (z. B. von Körperteilen). Dieser Funktionsunterschied ist aber so gut wie nie vorgeschlagen worden mit Ausnahme von Kammerzell (2000, 102). Ich unterstelle jedoch, dass hier nur eine Ungenauigkeit vorliegt und in Kammerzells gedrängter Darstellung die für „c. 2000 BC" gemachten Angaben sich eher schon auf einen neuägyptischen Sprachzustand beziehen sollen (vgl. auf S. 101: ,matters had been quite different in earlier varieties of Egyptian, and the organization of possessive constructions had been restructured several times“).

Dass von Alienabilität so wenig die Rede gewesen ist, kann nicht einfach an mangelnder linguistischer Bildung der Ägyptologen liegen. Wenn auch dieses Konzept früher nicht so populär war wie heute, spielt es doch bei den Possessivkonstruktionen des gesprochenen Arabisch, mit dem viele ältere Ägyptologen gut vertraut waren, eine zentrale Rolle. So stelle ich mir vor, dass manchen älteren Ägyptologen die Idee einer Erklärung mittels der Alienabilität gekommen sein dürfte, sie jedoch einen solchen Zusammenhang nicht erhärten konnten und daher nichts publizierten (vgl. Sander-Hansen 1936, 26 Anm. 1: „Sethe glaubte ursprünglich in Bezeichnungen für Gliedmaßen eine Gruppe mit regelmäßigem direkten Genitiv zu sehen und übertrug mir die genauere Nachprüfung“). Es zeigt sich nämlich bei einer näheren Betrachtung rasch, dass die Befunde des Älteren Ägyptisch mit einer solchen Funktionsunterscheidung nicht zu vereinbaren sind. Eher könnte man im Neuägyptischen, auf das ich hier nicht eingehe, nach der Alienabilität differenzierte unterschiedliche Possessivkonstruktionen erkennen, wenn auch mit Einschränkungen (vgl. dazu etwa Černý \& Groll 1984, 74-77; Depuydt 1999, 281 f.; Warburton 2015).

Die den älteren Forschern noch näherliegende semitistische Perspektive konnte dazu verleiten, einen $\mathrm{Zu}$ sammenhang mit der Determination zu suchen. Inspiriert von einer entsprechenden Regel im klassischen Arabisch, meinte Bakir (1966, 35f.) annehmen zu dürfen, dass „the first noun must be regarded as undefined when the indirect construction follows it; otherwise it must be defined“". Nach Grandet \& Mathieu (1990, 90 f.) soll hingegen die Definitheit des Rectums maßgeblich sein, und zwar sei dieses im direkten Genitiv indefinit, im indirekten Genitiv definit. Auch diese Ansätze erledigen sich bei einem näheren Blick auf die Belege sehr schnell. Weitere Arten von funktionalen Unterschieden, auf die ich hier nicht näher eingehe, wurden ganz beiläufig ohne detaillierte Argumentation von Junker (1938, 94, akzeptiert noch von Barta 1995, 30-32) und Junge $(1999,61)$ vermutet, und auch Faulkner (1973/8, III, 116 Anm. 16) muss eine irgendwie geartete funktionale Unterscheidung vorgeschwebt haben, wenn er zu einer Sargtextstelle kommentiert: „the direct genitive following [dem Wort 3 w.t, C.P.] may imply a gift intended for the god“".

Die funktionalen Differenzierungen mussten, damit sie auch nur halbwegs mit dem Material vereinbar waren, entweder sehr vage gefasst oder sehr großzügig ausgelegt werden. An dieser Stelle sei nur noch eine Meinung zur Verwendung des direkten Genitivs zitiert, die die Vagheit in besonders ausgeprägter Form zeigt: „Die ConstructusVerbindung ist dann anzuwenden wenn die enge Verbindung zweier Nomina angezeigt werden soll [...] Die Constructus-Verbindung kann auch gewählt werden, wenn das zweite Glied der Verbindung besonders betont werden soll“ (Spiess 1997, 68). So sind alle bisher vorgeschlagenen funktionalen Ansätze entweder mit den Belegen offensichtlich unvereinbar oder aber zu vage, damit nicht falsifizierbar und wissenschaftlich untauglich. Ich will sie hier nicht alle im Detail widerlegen, sondern stattdessen einen anderen Ansatz verfolgen, der hoffentlich auf Anhieb mehr überzeugt.

Dieser zweite, auch nicht eben neue Ansatz sieht einen Zusammenhang der Wahl der Genitivkonstruktion mit der als Regens verwendeten Vokabel oder mit deren grammatischer Form. Ich nenne dies die lexikalische Hypothese. So sah schon Erman (1928, §216), dass nach bestimmten Erstnomina der direkte Genitiv „besonders verwendet“ wird, darunter nach s.t „Ort“, $p r$ „Haus“, $n b$ „Herr“, z3 „Sohn“. Bald darauf und bislang am detailliertesten wurde die lexikalische Hypothese von SanderHansen (1936) ausgearbeitet. Er legt Statistiken zu Genitivverbindungen in den Pyramidentexten vor, die eine deutliche Abhängigkeit der Konstruktion vom Erstnomen zeigen, auch wenn er immer wieder einige Ausnahmen konstatieren musste. Um nur wenige seiner Ergebnisse hier zu referieren, steht der direkte Genitiv bevorzugt nach wj3 „Barke“ (S. 43), z3 „Sohn“ (S. 38), stj „Geruch“ (S. 45), smsw „Erstgeborener“ (S. 48) sowie generell nach fast allen Feminina, Pluralen und Dualen (S. 33), hingegen der indirekte Genitiv bevorzugt nach $j b$ „Herz“ (S. 35), wpwtj „Bote“ (S. 50), mw.t „Mutter“ (S. 51), $r$ „Mund“ (S. 31) und $k 3$ „Ka“ (S. 32). Vielleicht etwas vorschnell schlug SanderHansen denn auch gleich eine phonetische Interpretation als zugrundeliegende Ursache vor: „Der direkte Genitiv ist das Gewöhnliche nach allen Wörtern, die eine unbetonte Endung haben, im anderen Fall ist der indirekte Genitiv 
am häufigsten vertreten“ (S. 33). Sander-Hansens Entdeckung des lexikalischen Prinzips der Genitivverwendung ist ein wichtiges und bis heute nicht hinreichend gewürdigtes Verdienst. In seiner späteren Grammatik, die ja ein größeres Korpus als nur die Pyramidentexte behandelt, gibt er der Regel nur mehr eine sehr zurückhaltende Formulierung: „Es scheint überhaupt schwierig Regeln für die Erhaltung [des direkten Genitivs, C.P.] aufzustellen, nur scheint der direkte Genitiv bei Nomina, die mit einer Endung gebildet sind, häufiger und länger im Gebrauch“ (Sander-Hansen 1963, §210). Die lexikalische Hypothese hat sich zwar nicht wirklich als communis opinio durchgesetzt, wurde aber doch von einer Reihe von Forschern aufgegriffen. Sicher beeinflusst von Sander-Hansen stellt Edel (1955/64, §324) fest: „Der direkte Genetiv ist sehr häufig hinter Pluralen und Dualen“. Etwas vager glaubt Shisha-Halevy $(2007,240)$ an eine erhöhte Wahrscheinlichkeit für den indirekten Genitiv, wenn entweder das Erst- oder das Zweitnomen im Plural stehe, auch wenn oft, wie er zugibt, „the difference [...] is still obscure“.

Schenkel (1963) widmete dem Thema einen Aufsatz und legte umfangreiche neue Daten aus dem Alten und Mittleren Reich vor, die klar zeigen, dass mry „geliebt von“ häufiger mit dem direkten Genitiv konstruiert wird als ḩzy „gelobt von“. Seinen Belegstellen könnte man noch leicht weitere aus späterer Zeit hinzufügen wie etwa $m r y$ rmt.w, hzy $n$ ntr ,Geliebter der Menschen, Gelobter des Gottes“ (Urk IV 1218.3f.). Damit sehe ich in ihm ebenfalls einen wesentlichen Wegbereiter der lexikalischen Hypothese, auch wenn er selbst den Unterschied auf eine vermutete unterschiedliche Semantik beider Verben zurückführen und damit letztlich doch wieder auf eine funktionale Basis stellen wollte: „der direkte «Genitiv» drückt den Nahbereich aus, der indirekte den Fernbereich“ (S. 65). Kürzlich ist er darauf noch einmal zurückgekommen (Schenkel 2011, 70 f.) und hat seine alte semantische Erklärung als „allzu naiv“ verworfen. Nun vermutet er, dass es sich „bei den in Rede stehenden Verbindungen mit direktem Genitiv um relativ feste, komposita-artige Verbindungen“ handeln könnte, dass also mry $N$ als Kompositum aufgefasst wurde. Auch diese Erklärung ist noch nicht voll befriedigend, da zu zirkulär.

In einer neueren, einschlägigen Arbeit zum Thema von Jansen-Winkeln (2000) wird zwar betont, dass nicht nur eine einzige Ursache über die Wahl der Genitivkonstruktion entscheide, doch wird der lexikalischen Hypothese eine wesentliche Rolle eingeräumt: „offenbar haben bestimmte Wörter und Wortformen die Eigenschaft, als nomen regens einen der beiden Genetive zu implizieren. Das ist ganz offensichtlich bei den einsilbigen Maskulina (v. a. Namen von Körperteilen wie $j b, r$ ', $t p, h r,{ }^{C} w, r d$, aber auch $k$ 3, $r n$ u. a.m.), die in den Pyramidentexten im Singular das Rectum im indirekten Genetiv anschließen, im Dual und Plural aber im direkten.“ (Jansen-Winkeln 2000, 33). Umfangreiche diesbezügliche Wortstatistiken, allerdings für das Spätmittelägyptische, liefert er selbst in Jansen-Winkeln (1996, § 401). Ihm folgt - etwas zögernd auch Schweitzer (2005, 115): „Vielleicht sind damit die ägyptischen Nomina in zwei Gruppen zu unterteilen: zum einen gibt es Substantive, die als Regens auf eine Genitivverbindung beschränkt sind, zum anderen gibt es solche, die diese frei wählen können." Auch in neuesten Arbeiten spielt die lexikalische Hypothese weiter eine Rolle. Borghouts (2010, I, 296) bemerkt: „Direct annexion [...] is common with certain nouns as the head word, such as $n b$ lord, $s 3$ son, $p r$ house/ domain, $h w . t$ temple. “ Brose (2014, 110-117) stellt fest, dass bei bestimmten Erstnomina „primär die direkte Variante gewählt“ wird (u.a. $p r$ „Haus“), anderen Nomina hingegen „folgt bevorzugt ein indirekter Genitiv“ (u. a. h3 3 „Büro“). Schließlich bemerkt ScheeleSchweitzer $(2014,58)$ über aus Genitivverbindungen bestehende Personennamen des Alten Reiches, dass „auffällig ist, daß $h m$ stets mit direktem Genitiv und $b 3 k$ immer mit indirektem Genitiv konstruiert wird“ (beide Ausdrücke sind quasi-synonym: „Diener des ...“).

Angesichts der geschilderten Sachlage scheint mir die lexikalische Hypothese sehr attraktiv und jedenfalls gegenüber der funktionalen Hypothese vorzuziehen, und zwar aus zwei Gründen: Sie ist erstens besser mit den Belegen vereinbar und zweitens klarer formuliert, damit leichter falsifizierbar und wissenschaftlich höherwertig. Es wäre nun also für jedes Substantiv einzeln zu prüfen, welche Genitivkonstruktion es bevorzugt. Schon Schenkel $(1963,65)$ hielt es für „erforderlich [, d]ie Verteilung der «Genitive» innerhalb bestimmter Epochen zu katalogisieren“. Jansen-Winkeln $(2000,33)$ sagt, nachdem er den Einfluss des Erstlexems auf die Genitivkonstruktion dargelegt hat: „Diese Regeln im Einzelnen (und für die verschiedenen Epochen) zu entschlüsseln, würde umfangreiche Statistiken erfordern." Diese Forderungen sind heute immer noch ebenso aktuell wie vor 50 bzw. 15 Jahren.

In der Erwartung, dass die lexikalische Hypothese innerhalb eines möglichst homogenen Korpus besonders gut greifen sollte, habe ich mich entschlossen, sie anhand einer bestimmten Textgruppe, nämlich der Sargtexte (de Buck \& Gardiner 1935 ff., nur Bände 1-7), zu überprüfen. Die Sargtexte haben den Vorteil, ein großes, sprachlich wenigstens einigermaßen homogenes Korpus darzustellen. Da viele Texte mehrfach überliefert sind, lassen sich Individualfehler oft gut erkennen. Anderseits ist der Anteil an Fehlern und Nachlässigkeiten sehr hoch. Die Texte wurden im Akkord abgeschrieben, und Entstellungen des 
religiösen Inhalts hatten keine negativen Auswirkungen auf die Realwelt.

Man hätte leicht eine monographische Behandlung daraus machen können, wozu ich jedoch keine Absicht habe. Das Folgende soll nur eine Sondierung sein, die vielleicht andere Forscher zu weiteren Detailuntersuchungen ermuntern mag, darunter womöglich auch jüngere Ägyptologen, die nach einem Thema für ihre Abschlussarbeiten suchen. Ich bespreche für acht eher zufällig ausgewählte Vokabeln der Reihe nach, welche Wirkung sie als Erstnomen auf die gewählte Genitivkonstruktion haben. Ich will nicht behaupten, dass einzig und allein das Erstnomen über die Wahl der Genitivverbindung entscheidet, denn wie man sehen wird, bleibt immer ein Prozentsatz von Ausnahmen übrig. Es handelt sich aber zum mindesten um einen wesentlichen Faktor, den man als erstes herauspräparieren sollte. Die verbleibenden „Ausnahmen“ mögen dann durch das Einwirken weiterer Faktoren zu erklären sein, durch eine mögliche linguistische Inhomogenität des herangezogenen Korpus, oder eben teilweise doch durch Fehler.

Beim Aufsuchen der Genitivverbindungen hat mir ein Glossar gute Dienste geleistet, das ich aus einem Datensatz generiert habe, welchen mir Wolfgang Schenkel aus seiner in Vorbereitung befindlichen Sargtextkonkordanz zur Verfügung gestellt hat. Hierfür gilt ihm mein herzlicher Dank. Stellen, die zu stark zerstört, völlig evident korrupt oder ganz unverständlich sind, habe ich nicht berücksichtigt. Da ich beim Umgang mit problematischen Stellen vielleicht nicht immer völlig einheitlich verfahren bin sowie sich über manche Interpretation auch streiten lässt, sind alle im Folgenden gegebenen numerischen Angaben nur als Näherungen zu verstehen.

Zunächst einmal ist zu erkennen, dass in den Sargtexten der direkte Genitiv noch sehr häufig und produktiv ist. Die oben zitierten Äußerungen im Sinne einer eingeschränkten Verwendbarkeit des direkten Genitivs treffen auf die Sargtexte ganz und gar nicht zu. Auch wenn eine exakte Zahl schwer zu liefern ist, tritt nach meinem Eindruck der direkte Genitiv in diesem Korpus insgesamt häufiger auf als der indirekte. In fast allen einschlägigen Arbeiten wird erwähnt, dass der indirekte Genitiv stark bevorzugt wird oder nahezu obligatorisch ist, wenn das Erstnomen komplex, also seinerseits durch Attribute erweitert ist. Seltener, aber doch auch (Gardiner 1957, Nachtrag zu $\S 86$ auf S. 423; Barta 1995, 30; Jansen-Winkeln 2000, 33) wurde ein entsprechender Effekt für den Fall eines komplexen Zweitnomens behauptet. Wie wir sehen werden, wird die erstere Annahme in den Sargtexten bestätigt, die zweite hingegen nicht.
Nun gehe ich die acht Substantive der Reihe nach durch. Ich nenne bei allen Phrasen im Haupttext nur die Gesamtsumme der Textzeugen über alle Belegstellen hinweg und verlagere die Belegstellen selbst in die Fußnoten.

\section{$z^{3}$ „Sohn“}

Ich beginne mit Genitivgruppen, deren Regens aus dem Wort $z^{3}$ "Sohn“ besteht. Auf dieses folgt sehr häufig ein Genitiv, und zwar im Regelfall der direkte: $z 3 W_{s j r}$ „Sohn des Osiris“ $(29 \times)^{2}, z 3 G b$,Sohn des Geb“ $(23 x)^{3}, z 3 R^{c}$,Sohn des Re“ $(18 \times)^{4}, z 3$ nzr(.j) „Sohn eines zum $n z r$-Schrein gehörigen“ $(15 \times)^{5}, z 3$ wr „Sohn eines Großen“ $(14 \times)^{6}, z 3$ 3s.t „Sohn der Isis“ $(13 \times)^{7}, z 3$ Nw.t „Sohn der Nut“ $(12 \times)^{8}, z 3$ Jtmw „Sohn des Atum“ $(8 \times)^{9}, z 3 r p^{c} . t$ „Sohn des Fürsten“ $(5 \times)^{10}, z 3 j 3 w . t$,Sohn der Greisin“ $(3 \times)^{11}, z 3$ ntr „Sohn eines Gottes“ $(3 \times)^{12}, z 3$ sqd „Sohn des Seglers“ $(3 \times)^{13}, z 3$ Dh $h w t j$ „Sohn des Thot“ $(3 x)^{14}$, wozu noch verschiedene Streubelege in Form von direkten Genitiven mit seltener belegtem Zweitnomen kommen $(19 \times)^{15}$. Es liegt kein einziges sicheres Beispiel für eine indirekte Genitivverbindung aus $z 3$ und einem einfachen Nomen vor. Der einzige Kandidat wäre $z 3 n W_{s j r}$ „Sohn des Osiris“ $(1 \times)^{16}$, der jedoch aufgrund einer Zerstörung zweifelhaft bleiben muss.

Aber auch wenn das Zweitglied erweitert ist, ändert sich an dem Bild überhaupt nichts. Dies gilt zunächst noch wenig überraschend - für zusammengesetzte Götternamen, die man vielleicht als „erweitert" betrachten könnte: $z^{3}$ Hw.t-Hrw „Sohn der Hathor“ $(10 \times)^{17}, z 3 H r w$ 3h.tj „Sohn des Harachte“ $(5 \times)^{18}, z^{3} R^{c}$-Jtmw „Sohn des

2 CT I 194b, I 236h, II 69a, II 226a, IV 37f, IV 91e, V 231c, V 331j, V 3310, V 331t, V 331y, V 331dd, V 331ii, V 332d, VI 395j.

3 CT I 184b, I 233a, I 235f, II 40h, IV 93m, VI 215g, VI 392i, VII 467e.

4 CT IV 338l, IV 356b, IV 367d, V 240c, VI 161b, VI 197t, VI 278 h.

5 CT V 60c.

6 CT V 60c, VI 139b, VI $194 \mathrm{~g}$.

7 CT II 143b, II 226a, IV 37f, IV 86s, V 183e, VI 322i, VI 322j, VI 390d, VI 390h, VII 192k.

8 CT I 179m, V 23b, V 35h, VII 1n, VII 14n, VII 242b, VII 467e.

9 CT II 35i, II 44a, II 145b, IV 47f.

10 CT I $197 \mathrm{f}$.

11 CT VI 90a.

12 CT VI 84i, VI 84k, VI 85e.

13 CT V 66c.

14 CT III $127 \mathrm{~b}$, VI $278 \mathrm{~h}$.

15 CT III 58c, III 127b, III 176a, III 359a, IV 65i, IV 94m, VI 218j, VI 285g, VI 307i, VII 97s, VII 98i, VII 151a, VII 153k.

16 CT V $13 \mathrm{~b}$.

17 CT IV 9e, IV 179i, IV 181d, IV 182j, V 53g, VI 162v.

18 CT I 237b. 
Re-Atum“ $(3 \times)^{19}, z 3 \mathrm{Nfr}$-tm „Sohn des Nefertem“ $(2 \times)^{20}, z^{3}$ Nb.t-hw.t „Sohn der Nephthys“ ( $1 \times)^{21}$. Es gilt weiter für den Fall, dass das Zweitnomen um ein Suffixpronomen erweitert ist: $z 3 j t j=j$ „Sohn meines Vaters“ $(2 \times)^{22}, z^{3} z^{3}=k$, ,Sohn deines Sohnes“ $(2 \times)^{23}$. Man bleibt aber auch dann konsequent beim direkten Genitiv - und dies ist schon weniger selbstverständlich -, wenn das Zweitnomen durch ein Adjektiv erweitert ist: $z 3$ ntr ${ }^{c}$ "Sohn des großen Gottes“ $(3 \times)^{24}, z 3$ nb jry „Sohn von dessen Herrn“ $(2 \times)^{25}, z 33 z b$ wr „Sohn des großen Erleuchters(?)“ (1 $1 \times)^{26}$; wenn das Rectum seinerseits eine direkte Genitivverbindung ist: $z 3 n b p r$ „Sohn des Herrn des Hauses“ ( $9 \times)^{27}, z^{3}$ nb ntr.w „Sohn des Herrn der Götter“ $(6 \times)^{28}, z 3 s^{c} h R^{c}$ „Sohn eines Edelmanns des Re“ $(6 \times)^{29}, z 3$ b3.w Jwnw „Sohn der Seelen von Heliopolis“ $(2 \times)^{30}, z^{3} \mathrm{nb} \mathrm{tm}$ „Sohn des Allherrn“ $(2 \times)^{31}$; (syntaktisch etwas anders einzuordnen:) $z 3$ wb3.t $t 3$, ,Sohn der Erdöffnerin“ $(2 x)^{32}$; sogar wenn das Rectum eine indirekte Genitivverbindung ist: $z 3$ dry.t n.t Wsjr „Sohn der Weihe (Raubvogel) des Osiris“ $(6 \times)^{33}$, oder wenn es anderweitig etwas länger ist: $z 3$ Jhs.t jmj.t $n 3 w$, „Sohn der Ihset, die im Feuer(?) ist“ $(2 \times)^{34}$. Gesondert zu erwähnen sind noch Genitive mit dem Eigennamen des Toten (NN) als Zweitglied. Dieser wurde bekanntlich aus einem Pronomen der Textvorlage hergestellt, manchmal in recht mechanischer Weise (dazu vgl. de Buck 1949, 89 und Doret 1989, 46). Das einzige verfügbare Beispiel mit $z 3$ zeigt auch wieder den direkten Genitiv: $z 3 N N$ tn „Sohn dieser NN“ (1× $)^{35}$.

Ist jedoch das Erstnomen durch ein Attribut erweitert, so wird in der Regel der indirekte Genitiv benutzt. Dies gilt für eine Erweiterung durch ein Demonstrativum: $z^{3} \mathrm{pn} / \mathrm{pw}$ $n$ 3s.t „dieser Sohn der Isis“ $(10 \times)^{36}, z 3$ pw n msi.t Jtmw „dieser Sohn derjenigen, die den Atum gebar“ $(4 \times)^{37}, z^{3} p w$ $n N w . t$,dieser Sohn der Nut“ $(1 \times)^{38}, z^{3} p w n R^{c}-J t m w$,die-

19 CT II 35i, II 145b, VI $197 \mathrm{~g}$.

20 CT IV 48a.

21 CT IV $180 \mathrm{~b}$.

22 CT IV $55 i$.

23 CT I 231e.

24 CT III 58d.

25 CT IV 96j, IV 338b.

26 CT VII $215 \mathrm{e}$.

27 CT I 111b.

28 CT I 228b, I 232d.

29 CT VII 279b.

30 CT III 194c.

31 CT VI 134g.

32 CT VI 3d.

33 CT VII 269a.

34 CT V 397a.

35 CT VI 157j; ein anderer Textzeuge hat hier $z 3=j$ „mein Sohn“.

36 CT II 350c, VII 191a.

37 CT III 384d.

38 CT V 233b. ser Sohn des Re-Atum“ (1× ${ }^{39}$; oder für eine Erweiterung durch ein Adjektiv: $z 3$ smsw $n$ Jtmw „erstgeborener Sohn des Atum“ $(2 \times)^{40}, z 3 \underline{d p} . j n$ Wsjr ,erster Sohn des Osiris“ $(2 \times)^{41}, z 3 \underline{d p}$.j $n R^{c}$,erster Sohn des Re“ $(1 \times)^{42}, z 3$ wr $n G b$ „ältester Sohn des Geb“ (1× $)^{43}$. Zwei Ausnahmen mit einem direkten Genitiv trotz Attribut beim Erstnomen stehen so vereinzelt, dass die Annahme eines Fehlers oder einer orthographischen Ungenauigkeit möglich erscheint: $z 3$ pn 3s.t „dieser Sohn der Isis“ (1× $)^{44}, z 3$ pw smsw Nw.t ,dieser erstgeborene Sohn der Nut“ $(1 \times)^{45}$. Wohl echt ist hingegen der direkte Genitiv im Falle einer Koordination des Erstglieds: $z 3$ z3.t Nw.t „Sohn und Tochter der Nut“ $(2 \times)^{46}$. Ein interessanter Sonderfall ist auch $z 3$ js Hnn $(3 \times)^{47}$, wo vermutlich die archaische Postposition $j s$ vorliegt: „wie der Sohn des Henen“.

Im Ägyptischen besteht aber auch die Möglichkeit, ein auf das Erstnomen bezügliches Attribut hinter die gesamte Genitivverbindung zu stellen, die in diesem Fall direkt sein muss. In den Sargtexten ist dafür kein ganz zwingender Beleg mit $z 3$ „Sohn“ greifbar. Ein Kandidat wäre die folgende, syntaktisch ambige Stelle: $z 3 j t j=f j q r$,der Sohn seines ehrwürdigen Vaters“ „der ehrwürdige Sohn seines Vaters“ “ $(2 \times)^{48}$, wo sich auch die Interpretatoren uneins sind: "the son of my worthy father" (Faulkner 1973/8, II, 113) „le fils excellent de son père“ (Carrier 2004, $1153 \mathrm{f}$.).

\section{pr „Haus“}

Als nächstes komme ich zu dem Substantiv $p r$ „Haus“ als Erstnomen von Genitivverbindungen. Um das Ergebnis vorwegzunehmen, vermute ich, dass es sich ebenso verhält wie $z^{3}$ „Sohn“. Ist kein Glied erweitert (von hier an betrachte ich auch zusammengesetzte Götternamen und Substantive mit Suffixpronomen im Rectum als unerweitert), so steht also auch $p r$ wieder konsequent mit dem direkten Genitiv: $p r$ Wsjr „Haus des Osiris“ $(27 \times)^{49}, p r$ Dhwtj „Haus des Thot“ (13x) ${ }^{50}$, pr Rw.tj „Haus des Ruti“

39 CT VII $97 \mathrm{k}$.

40 CT VI 281t (dort Jtmw verderbt zu jmtw), VII $111 \mathrm{t}$.

41 CT V 12e.

42 CT IV 180a.

43 CT I 178 b.

44 CT II 350c.

45 CT V 12 e.

46 CT II $18 \mathrm{~g}$.

47 CT III $86 \mathrm{~b}$.

48 CT VI 20o, VI $21 \mathrm{~g}$.

49 CT II 197c, II 294b, III 311a, IV 81g, IV 96a, IV 96b, IV 96c, IV 96e, IV 96i, IV 307a, IV 339a, IV 370b.

50 CT II 93f, II 95g, III 1c, III 75f, III 105d, III 137a, VI 305j. 
$(11 \times)^{51}, p r$ Hrw „Haus des Horus“ $(10 \times)^{52}, p r$ Spd.t „Haus der Sothis“ $(9 \times)^{53}, p^{3} 3^{3} . t$, Haus der Maat“ $(8 \times)^{54}$, pr b3.wj „Haus der beiden Bas“ $(7 \times)^{55}$, pr hwr.t „Haus der Elenden“ $(6 \times)^{56}, p r$ ntr „Haus des Gottes“ $(4 \times)^{57}, \mathrm{pr} 3$ s.t „Haus der Isis“ $(3 \times)^{58}, \mathrm{pr} \check{S} w$ „Haus des Schu“ $(3 \times)^{59}, \mathrm{pr}$ Hntj-mnj. $t=f$ „Haus des Chentimenitef“ $(3 \times)^{60}, p r k k w$ „Haus der Dunkelheit“ $(3 \times)^{61}$; dazu kommen eine Reihe von Streubelegen $(27 \times)^{62}$. In $p r n \underline{d . t} t=k(2 \times)^{63}$ denken die bisherigen Übersetzer an das Substantiv d.t „Totenstiftung“ (z. B. Barguet 1986, 179 „dans ton domaine funéraire“), womit ausnahmsweise ein indirekter Genitiv stehen würde. Ich möchte hier lieber den grammatikalisierten Ausdruck $n$ $\underline{d} . t=f$,sein eigener" annehmen (Hannig 2006, 2806) ebenso wie in dem vergleichbaren $p r=f n \underline{d} . t=f$, sein eigenes Haus“ $(4 \times)^{64}$.

Auch hier gilt wieder, dass die Erweiterung des Zweitgliedes nicht zu einem indirekten Genitiv führt: $p r$ b $p f$ „Haus jenes Bas“ $(4 \times)^{65}$ (grammatisch wäre auch „jenes Haus des Bas“ denkbar, aber die angenommene Klammerung wird unterstützt durch das morphologisch eindeutige hw.t b3 pf „Haus jenes Bas“ in CT I 273c), pr b3 pn „Haus dieses Bas“ $(1 \times)^{66}$ (vgl. den vorstehenden Fall), $p r$ $j t j=f$ Jtmw „Haus seines Vaters Atum“ $(3 \times)^{67}, p r m \underline{d} 3$.t nt $\underline{\text { r }}$ „Haus des Gottesbuches“ $(2 \times)^{68}$, pr Wsjr $n b \underline{D} d w$ „Haus des Osiris, Herrn von Busiris“ (1× $)^{69}$, pr Hrw Dh $h w t j$ „Haus des Horus und Thot“ $(1 \times)^{70}$. Wahrscheinlich irrelevant ist in diesem Zusammenhang pr Wsjr $n$ D $d w$,Haus des Osiris von Busiris“ $(8 \times)^{71}$, da dieses eher als $\left[p r W_{s j r}\right] n \underline{D} d w$ zu klammern ist. In CT V $2 \mathrm{~b}$ wurde das originale $p r=j p w$

51 CT III 327b, IV 81f, V 47a.

52 CT III 1c, III 75f, III 105d, III 137a, III 137d, III 151e, V 47b, VII 226k, VII 231g.

53 CT I 143a, I 146c.

54 CT VII 371j.

55 CT I 226a, V 395f, VI 251b, VI 340j.

56 CT V 283c.

57 CT III 75h, III 197 e.

58 CT I 268j.

59 CT III 40g, V 34a,

60 CT II 258a.

61 CT IV 70a.

62 CT I 294g, III 1c, IV 28k, IV 65d, V 18b, V 34a, V 47b, V 67c, VI 174c, VI 195d, VI 195g, VI 197o, VI 260k, VI 278q, VI 288j, VI 301o, VI 339c, VII 20a, VII 96f, VII 971, VII 163j, VII 2261, VII 231g, VII 304j.

63 CT I $164 f$.

64 CT V 243c.

65 CT I 284i, VI 401p.

66 CT II 291e.

67 CT IV 207a.

68 CT VII 104 f.

69 CT III 311a.

70 CT VI 287 e.

71 CT II $290 \mathrm{~b}$. „dieses mein Haus“ z. T. umgesetzt einerseits in das grammatisch korrekte $p r N N$ tn „das Haus dieser NN“ (2x), andererseits in das etwas gewagte $p r p w N N p n(1 \times)$,dieses Haus dieses NN“, woraus man jedoch kaum viel wird ableiten können.

Ist das Erstglied erweitert, so tritt der indirekte Genitiv ein: $p r$ wr $n$ Hw.t-Hrw „das große Haus der Hathor“ $(3 \times)^{72}$, $p r=j / f n c h h . w$, ,mein/sein Haus der Lebenden“ $(3 \times)^{73}, p r=k$ $n^{c} n h$, dein Haus des Lebens“ $(2 \times)^{74}, p r=k n 3 w . t-j b$,dein Haus der Freude“ $(2 \times)^{75}$. An der letztgenannten Textstelle schreibt ein Textvertreter $\mathrm{pr} n$ 3w. $t$-jb ,?das Haus der Freude" ohne Suffix, wo der indirekte Genitiv gemäß meinen Regeln unerwartet wäre, doch kann diese Lesart verderbt sein. Ein als pr pn Djgg „dieses Haus des 'Digeg'“ $(1 \times)^{76}$ gelesener Beleg würde meiner Regel widersprechen, wobei neben der anstößigen Grammatik noch ein sonst unbelegter Gottesname anzunehmen wäre. Ich emendiere zu pr pn $n$ Jgg mit demselben Schlangengott Jgg, der schon kurz vorher in demselben Text auftritt (CT VII 95g); im hieratischen Text könnte das $n$ in ein $d$ verschrieben worden sein.

Wie schon gesagt, kann die Genitivkonstruktion $\mathrm{N}_{1}-\mathrm{N}_{2}$ in dem Fall, dass zum Erstnomen ein Attribut tritt, entweder zu $\mathrm{N}_{1}-$ Attr $_{1}$-nota genitivi- $\mathrm{N}_{2}$ oder zu $\mathrm{N}_{1}-\mathrm{N}_{2}-\mathrm{Attr}_{1}$ expandiert werden. Die zweite Variante ist in den Sargtexten schwächer belegt, liegt aber eindeutig vor in $p r \check{s} n^{c} p w$ „dieses Arbeitshaus“ (3x) $)^{77}$.

\section{$n b$,Herr“}

Als nächstes komme ich zu dem Substantiv $n b$ „Herr“, welches aufgrund seiner Bedeutung überaus häufig im Erstglied einer Genitivverbindung auftritt. Auch dieses verlangt im Regelfall den direkten Genitiv: $n b \mathrm{tm}$ „Herr des Alls“ $(59 \times)^{78}, n b m^{3}{ }^{c} . t$ „Herr der Maat“ $(55 \times)^{79}, n b$ ntr:w

72 CT VI 130g.

73 CT I $168 \mathrm{c}$.

74 CT I $243 \mathrm{~d}$.

75 CT I $242 b$.

76 CT VII 97 f. Nach freundlicher Mitteilung von Harco Willems (Leuven), der mir eine Photographie der Stelle übermittelte, scheint das $d$ paläographisch sicher.

77 CT V 150 c.

78 CT I 20c, I 194g, I 195c, I 202b, I 211a, I 246l, II 101b, III 27b, III 260c, III 265b, III 353a, III 382b, IV 61m, IV 84l, IV 85f, IV 391c, V 273d, V 285a, VI 131j, VI 134g, VI 400t, VII 15n, VII 181c, VII 271a, VII 321b. 79 CT I 53c, I 352d, IV 170c, IV 254a, VI 162q, VI 267b, VI 267o, VI 347e, VII 133p, VII 204i, VII 268a, VII 279c. 
„Herr der Götter“ $(48 \times)^{80}$, nb jmn.t „Herr des Westens“ $(47 \times)^{81}, n b$ 3h.t „Herr des Horizonts“ (38× $)^{82}, n b^{c} n h$ „Herr des Lebens“ $(33 \times)^{83}, n b$ nhh „Herr der Ewigkeit“ $(27 \times)^{84}$, $n b$ ps $\underline{d}$.(w) $t$ „Herr der Neunheit(en)“ $(26 \times)^{85}, n b \underline{d}$.t „Herr der Ewigkeit“ $(25 \times)^{86}, n b s p$ 3. (w) $t$ „Herr des Gaues/der Gaue“ $(23 \times)^{87}, n b$ wsrw(.t) „Herr der Macht“ nb wsr.tjw „Herr der Mächtigen“ $(22 \times)^{88}, n b$ B $3 \underline{h} w$ „Herr von Bachu“ $(22 \times)^{89}, n b$ 3h.w. „Herr der Totengeister“ $(19 \times)^{90}, n b$ hnkk.(w) $t$ „Herr der Locke(n)/der Gelockten“ (19x $)^{91}$, nb p.t „Herr des Himmels“ $(17 \times)^{92}, n b$ htp.(w) $t$ „Herr des Opfers/der Opfer“ $(16 \times)^{93}, n b$ dšr.w „Herr der roten Farbe (des Blutes/des Morgenrots)“ (16x $)^{94}, n b$ jm 3 h „Herr der Versorgtheit“ (14× $)^{95}, n b \operatorname{pr}(=j)$ „Herr des/meines Hauses“ $(14 \times)^{96}$, $n b$ sh. $(w) t$ „Herr des Feldes/der Felder“ $(14 \times)^{97}, n b p^{c} . t$ „Herr der Bürger“ $(13 \times)^{98}, n b \underline{t}{ }^{3} w$ „Herr der Luft“ $(13 \times)^{99}$, $n b$ nrw „Herr des Schreckens“ $(12 \times)^{100}, n b j 3 h w$ „Herr des Sonnenlichts“ $(11 \times)^{101}, n b$ gmw.t „Herr der Schwäche“ $(11 \times)^{102}, n b$ 3.t „Herr der Kraft/des Augenblicks“ $(10 \times)^{103}$,

80 CT I 202c, I 215d, I 219c, I 223g, I 228b, I 239e, I 243g, I 357e, I 357f, II 214c, II 219b, IV 144g, IV 145e, IV 147p, IV 181k, IV 181l, V 257d, V $333 \mathrm{k}, \mathrm{V} 377 \mathrm{c}$.

81 CT I 183e, I 189f, I 192h, I 205b, I 234g, I 237b, I 245m, II 151e, III 320d, IV 90e, V 125a, V 329c, VI 218q, VI 232j, VI 243k, VI 263f, VI 301 e. 82 CT I 201a, I 236d, IV 114f, V 8d, VI 153c, VI 235k, VII 1r, VII 44i, VII 306a, VII 400a, VII 469e, VII 500b.

83 CT I 2440, I 255a, I 393b, I 394h, II 387a, IV 72g, IV 342c, V 297c, VI 1311, VI 267k, VI 269q, VI 269s, VI 269u, VI 379l, VI 3790.

84 CT II 22a, IV 145a, V 293d, V 301c, V 312d, V 313e, VI 224t, VI 340 i, VII 10j, VII 465e, VII 468c, VII 485b.

85 CT I 230b, IV 37d, IV 99e, IV 99f, IV 190a, IV 310a, IV 330g, VI $269 \mathrm{~m}$.

86 CT I 201h, II 39b, IV 111b, IV 123a, IV 143d, V 294a, V 298a, V 302c, VII 1t, VII 10l, VII 28j.

87 CT IV 259a, IV 264a, VII 112j.

88 CT II 129a, VII 150a, VII 279a, VII 337b.

89 CT II 377a, II 387a, IV 46k.

90 CT IV 18e, VI 274c, VII 293a, VII 501a.

91 CT III 195i, VI 263k, VII 18i, VII 220e, VII 231f, VII 277 a.

92 CT II 401b, III 318h, V 165c, VI 114c, VI 218p, VII 1t, VII 112n, VII $112 \mathrm{u}$.

93 CT III 298d, III 309g, IV 115d, V 382j, VI 96e, VI 237r, VI 258b, VI 274b, VI 347j, VII 3f, VII $27 \mathrm{~g}$.

94 CT IV 181i, IV 316c, IV 328i, V 188e, V 188f, V 257g, VI 193d, VII 355d.

95 CT III 219e, IV 3261, IV 326n, IV 342c, V 202h, V 203a, V 208o, VI $414 \mathrm{u}$, VII 27o, VII $112 \mathrm{u}$, VII $127 \mathrm{u}$, VII $128 \mathrm{c}$.

96 CT I 111b, V $400 f$.

97 CT IV 2g, V 348b, V 353:iv, V 368b, V 371a, V 385a.

98 CT I 54e, I 81o, I 305a, VI $125 f$.

99 CT II 402a, III 306d, III 311g, V 369c, VI 252g, VII 469b.

100 CT II 137g, IV 182c, IV 316b, IV 316c, IV 328h, VI $77 \mathrm{i}$.

101 CT IV 159a, VI 150i, VII 17c, VII 214c.

102 CT I 43b, I 268e.

103 CT VI 305g, VII 334d. $n b$ h.t „Herr von Besitz“ $(10 \times)^{104}, n b$ sj3 „Herr der Weisheit“ $(10 \times)^{105}, n b 3 b d w$,Herr von Abydos“ $(9 \times)^{106}, n b$ nšnj „Herr der Wut“ $(9 \times)^{107}, n b t^{3}$ „Herr der Erde“ $(9 \times)^{108}, n b$ wrr.t $(=f)$ „Herr der/seiner Wereret-Krone“ $(8 \times)^{109}$, , $b$ rhy.t „Herr der Untertanen“ $(8 \times)^{110}, n b$ Himnw „Herr von Hermopolis“ $(7 \times)^{111}, n b$ Sp 3 „Herr von Sepa“ $(7 \times)^{112}, n b$ shm.w „Herr der Szepter“ $(7 \times)^{113}, n b$ sšp „Herr des Lichts“ $(7 \times)^{114}$, $n b$ wtnw „Herr der Flut“ $(6 \times)^{115}, n b$ nbj „Herr der Flamme“ $(6 \times)^{116}, n b h w$,Herr des Befehls“ $\left.{ }^{(6 \times}\right)^{117}, n b j^{c} r . t j / j{ }^{c} r . w t$ „Herr der (beiden) Uräen“ $(5 \times)^{118}, n b$ jmj.wt „Herr der darin befindlichen(?)“ $(5 \times)^{119}, n b$ w3h „Herr der Vegetation“ $(5 \times)^{120}, n b$ phtj „Herr der Kraft“ $(5 \times)^{121}, n b$ f3w „Herr der Pracht“ $(5 \times)^{122}, n b R-m 3 q w$ „Herr von Ramaqu“ $(5 \times)^{123}, n b$ $h n w$ „Herr der Henu-Barke“ $(5 \times)^{124}, n b$ h $\underline{d} . t(=f)$ „Herr der/ seiner weißen Krone“ $(5 \times)^{125}$, nb qrs.t „Herr des Begräbnisses“ $(5 \times)^{126}, n b$ k 3.w ,Herr der Kas“ $(5 \times)^{127}, n b$ t3.wj $(=f j)$ „Herr der/seiner beiden Länder“ $(5 \times)^{128}, n b$ dw3.t „Herr der Unterwelt“ $(5 \times)^{129}, n b$ D pw „Herr von Dep“ $(5 \times)^{130}, n b$ $\underline{d b . w}$ „Herr der Einkünfte(?)“ $(5 \times)^{131}, n b \underline{d s r}$.wt „Herr der Heiligkeit“ $(5 \times)^{132}$. Einige dieser Belege sind nicht vollkommen hieb- und stichfest: So könnte man statt $n b^{c_{n h}}$,Herr des Lebens“, $n b$ sj3 „Herr der Weisheit“ und $n b$ nšnj „Herr der Wut“ rein grammatisch auch „lebender Herr“, „weiser Herr“ bzw. „wütender Herr“ lesen. Trotzdem ist die Beleglage für den direkten Genitiv nach $n b$ überwältigend. $\mathrm{Zu}$

104 CT IV 310a, VII 455b.

105 CT VII 274b.

106 CT I 179e, III 308b, V 194h, V $207 i$.

107 CT VII 263b.

108 CT II 401b.

109 CT VI 99c, VI 268t.

110 CT V 129b, VI $22 f$.

111 CT II 148c, III $241 \mathrm{~b}$.

112 CT III 265a, III 315d, VI 2131.

113 CT II 402a, VII $193 i$.

114 CT IV 620, VII 294a.

115 CT IV 144d, IV 144h, V 217e, VI 86a, VI 319k.

116 CT II 10b, V $302 \mathrm{~b}$.

117 CT IV $157 \mathrm{~b}$, VI $265 \mathrm{~h}$.

118 CT IV 80g, VI 300n, VII 268a.

119 CT VII 269b.

120 CT V 374a.

121 CT VI 253o, VI 254c, VI 254t, VII 96u, VII $235 f$.

122 CT V 217b, V $217 d$.

123 CT V $125 b$.

124 CT V $124 \mathrm{c}$.

125 CT I 217a, VI 99c.

126 CT VI 94h, VI 296c, VI 351e, VII 12p, VII 137a.

127 CT II 162a, III 262d, III 388b.

128 CT I 305b, VII 30 o.

129 CT V 330i, VI 184g, VII 112h.

130 CT V $128 \mathrm{~b}$.

131 CT VII 298a.

132 CT V 375d. 
diesen explizit aufgeführten Verbindungen kommt noch eine große Zahl von Streubelegen (ca. 200×), für die ich auf Nachweise verzichte.

Auch wenn das Zweitglied erweitert ist, bleibt es nach wie vor beim direkten Genitiv: $n b$ h.t 7 „Herr von sieben Portionen“ (auch mit anderen Zahlwörtern) $(27 \times)^{133}, n b$ h.t 5 jptw ${ }^{\text {3.t }}$ „Herr dieser fünf großen Portionen“ $(2 \times)^{134}, n b$ sh.wt w3 $\underline{d} . w t$ „Herr grüner Felder“ $(13 \times)^{135}, n b$ sh.t(j) htp „Herr des Opferfeldes/der beiden Opferfelder“ $(11 \times)^{136}$, nb mrw.tj jpt.j „Herr dieser beiden Freundinnen“ (10× $)^{137}$, $n b$ c $3 b . t$ wr.t „Herr des großen Opfers“ $(6 \times)^{138}, n b$ t3 $\underline{d} s r$ „Herr des heiligen Landes“ $(6 \times)^{139}, n b$ h $h w . t c^{c} \check{S}^{2} m w$ „Herr des Hauses der Kultbilder“ $(4 \times)^{140}, n b h r w^{c}$ „Herr eines Gesichts“ $(4 \times)^{141}$, nb mtw.t 5 k3.w „Besitzer des Samens von fünf Stieren“ “ $(3 \times)^{142}, n b$ hw.t ${ }^{c} 3 . t$,Herr des großen Hauses“ $(2 \times)^{143}, n b$ h w.t d šr.t jmj.t 3 h.t „ „Herr des roten Hauses, das im Horizont ist“ $(2 \times)^{144}$, nb sh.t j3r.w „Herr des Binsengefildes“ $(2 \times)^{145}, n b$ sh.t $t n$ „Herr dieses Feldes“ $(2 \times)^{146}, n b$ dpj rnp.t „Herr des Jahresbeginns“ $(2 \times)^{147}$, nb 3.t wr.t „Herr der großen Kraft“ $(1 \times)^{148}$, nb wrr.t wr.t „Herr der großen Wereret-Krone“ $(1 \times)^{149}$, nb p.t mhtj.t „Herr des nördlichen Himmels“ (1× $)^{150}, n b$ pr.t-hrw „Herr des Anrufungsopfers“ $(1 \times)^{151}, n b$ n'.t wr.t „Herr der großen Stadt“ $(1 \times)^{152}, n b$ Nnj$n z w$ „Herr von Herakleopolis“ $(1 \times)^{153}, n b$ h hw.tj jmnt.t „Herr der beiden westlichen Häuser“ $(1 \times)^{154}, n b z m^{3} t^{3}$ „Herr der Vereinigung mit der Erde“ $(1 \times)^{155}, n b$ t3 $c_{n h . w}$.Herr des Landes der Lebenden“ $(1 \times)^{156}$.

133 CT III 1b, III 75f, III 78c, III 125g, III 161c, III 166d, III 176b, VI 197j, VI 198q, VI 208f, VI 288h, VII $231 \mathrm{~g}$.

134 CT III $99 f$.

135 CT I 346c.

136 CT V 336a, V 348b, V 351d, V 352f, VI 290a, VI 290 e.

137 CT V 293e, V 297e, V 301d.

138 CT III 176a, III 198e.

139 CT V 165a, VI $142 \mathrm{~m}$.

140 CT II 218b.

141 CT VI 322ee, VI 323f, VI 323k, VI 323p.

142 CT V 221a.

143 CT IV 61n, IV 311c.

144 CT VII 495h.

145 CT II $151 \mathrm{~b}$.

146 CT V 353:iv.

147 CT IV 51h.

148 CT VII $250 f$.

149 CT VII $148 \mathrm{i}$.

150 CT III 138b.

151 CT VII 137a.

152 CT VI 286p.

153 CT V 257d.

154 CT VII 1880.

155 CT VII 137a.

156 CT VII $27 \mathrm{v}$.
Dies gilt auch für die Ausdrücke $n b \check{s} n h$ 3 , \#Herr des Sees des Gemetzels“ $(3 \times)^{157}$ und $n b$ š. $w$ h 3 , \#Herr der Seen des Gemetzels“ ( $(1 \times)^{158}$, die noch eine kurze Diskussion erfordern. Im Rectum des ersteren sehe ich wie schon andere (Altenmüller 1966; Luft 1978, 35 f.; Krauss 1997, 14-17) eine indirekte Genitivverbindung, da der Beleg im Plural den entsprechenden direkten Genitiv zeigt. Die Korrespondenz, dass auf einen Singular der indirekte und auf einen Plural der direkte Genitiv folgt, ist für einige Substantive typisch, wie ich unten darlegen werde. Eine andere Fraktion von Ägyptologen (z. B. Sethe 1935 ff., II, 44; van der Molen 2000, 240; Hannig 2006, 2977; unentschlossen ist Wb III 222.12,13) segmentiert den Ausdruck als š $n h$ 3 "gewundener See“, doch ist dieses vermeintliche Adjektiv sonst nicht bekannt.

Nach $n b$ ist aber auch der indirekte Genitiv nachweisbar. Den besten Beleg dafür liefert die Phrase $n b n n t j . w$ „Herr der Seienden“ $(5 \times)^{159}$; nur ein Textzeuge schreibt $n b n t j . w$. Das Rectum besteht hier aus einem substantivierten Adjektiv. Ich kann mir hierzu folgende Erklärung vorstellen: In der Abfolge Substantiv-Adjektiv bildet das letztere normalerweise ein adjektivisches Attribut zum Substantiv. Wenn es aber in einer genitivischen Rolle verstanden werden soll, so konnte dies durch die nota genitivi verdeutlicht werden. Im vorliegenden Fall hätte zwar das Pluralmerkmal eigentlich zur Klarstellung der Syntax ausreichen sollen, aber offenbar mochte man sich allein darauf nicht verlassen, womöglich weil die Kongruenz der Adjektive in der gesprochenen Sprache nicht immer eindeutig wahrnehmbar war. Es stehen noch zwei weitere Beispiele für dieselbe Situation zur Verfügung: $n b$ n jmj.w s.t - $^{C}$ "Herr derer, die in der Tätigkeit sind“ $(2 \times)^{160}$ mit der nota genitivi zwischen $n b$ und einer substantivierten Nis-

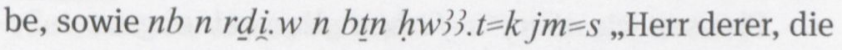
dem gegeben wurden, der deine Verwesung in ihr ablehnt (o. ä.)“ ( $(1 \times)^{161}$ mit der nota genitivi zwischen $n b$ und einem substantivierten Partizip. Wie ich meine, ist also nicht ein mechanisches syntaktisches Kriterium wie etwa die Länge oder Komplexität des Rectums für die Verwendung des indirekten Genitivs verantwortlich, sondern die eher weiche und semantische Eigenschaft, dass ansonsten aus der Sicht der Ägypter die Gefahr einer Ambiguität bestünde. Dies kann leicht dann der Fall sein, wenn ein substantiviertes Adjektiv als Rectum fungiert.

\footnotetext{
157 CT IV 1a, IV 36a.

158 CT IV 35a.

159 CT I 199b.

160 CT VI 178n.

161 CT VII 472b.
} 
Einen anderen interessanten Beleg für den indirekten Genitiv nach $n b$ finden wir in folgender Stelle: jnk $n b n$ 3h.wt $m$ j3.t wr.t, $n$ qrs $m$ jmn.t $n$ fr.t , ich bin ein Besitzer von Äckern auf dem großen Hügel und eines Grabes im schönen Westen“ (2×, davon $1 \times$ am Schluss verderbt $)^{162}$. Hier regiert $n b$ zwei koordinierte Genitive, und die syntaktische Struktur des Ganzen wäre ohne nota genitivi wohl nur schwer durchschaubar. Ich vermute auch hier, dass das Setzen des $n$ nicht primär durch die Länge des Rectums verursacht ist, sondern zum Zweck der Vermeidung von Ambiguität in dieser speziellen Situation.

Nur ganz sporadisch können wir nach $n b$ einen indirekten Genitiv außerhalb dieser Bedingungen registrieren, und zwar als Variante von Phrasen, die (siehe oben) anderswo die direkte Konstruktion haben: $n b n$ wtnw „Herr der Flut“ $(1 \times)^{163}$, nb $n$ h.t 7 „Herr von sieben Portionen“ $(1 \times)^{164}, n b n$ ntr.w „Herr der Götter“ $(1 \times)^{165}$ (die Stelle ist wegen überschüssiger Pluralstriche etwas dubios). Diese wenigen Belege muss ich als unerklärte Einzelfälle auf sich beruhen lassen.

In CT I 358b schreiben 3 Textzeugen $s^{c} r$ p.wt nb.t $n$ $n t r . w$ jmj.w zn.w=sn ,qui fait monter tous les cieux aux dieux qui sont dans leur portes“ (Barguet 1986, 464), was ich für den Urtext halte. Da in anderen Textzeugen die Kongruenz von $n b$ unterbleibt, was nicht ungewöhnlich ist (Sperveslage 2010, 230 Anm. 45), konnte eine Variante mit $s^{c} r$ p.t $n b \quad n$ ntr.w ... entstehen, die wie ein indirekter Genitiv nach dem Wort „Herr“ erscheinen mochte. Ein Textzeuge hat dies in der Tat eindeutig so verstanden ( $n b$ mit Gottesklassifikator geschrieben). Vier Textzeugen haben dagegen das $n$ gestrichen, so dass sich wieder ein regulärer direkter Genitiv nach (dem vermeintlichen) „Herr“ ergibt.

Phrasen wie $n b n j$ š. $t=f n b(. t)$ „Herr seines ganzen Besitzes“ (Wb II 228.9, in CT nicht belegt) haben den indirekten Genitiv also wohl nicht nur, wie angenommen wurde (Jansen-Winkeln 2000, 33), weil das Zweitglied komplex ist. Ich vermute auch hier als Ursache, dass eine Phrase ${ }^{\star} n b j$ š. $t=f n b$ zu ambig gewesen wäre, da sie sowohl „Herr seines ganzen Besitzes“ als auch ,jeder Herr seines Besitzes“ hätte bedeuten können.

Eine Konstruktion mit erweitertem Erstnomen, die analog zu den oben behandelten Substantiven sicherlich den indirekten Genitiv erfordern würde, sollte auch von $n b$ möglich gewesen sein (z. B. * $n b$ pn $n X$, dieser Herr des

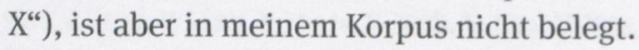

162 CT II 125i-126a.

163 CT IV 145a.

164 CT VII 226j.

165 CT I 219c.

\section{w3.t "Weg"}

Genitivverbindungen mit w3.t „Weg“ im Regens werden, wie wohl generell mit den meisten femininen Substantiva (was aber im Detail noch nachzuweisen bleibt), ebenfalls üblicherweise direkt konstruiert: $w 3 . t{ }^{3}{ }^{3}$. $t$, Weg der Maat “ $(12 \times)^{166}$, w3.t $N(n) w$,Weg des Nun“ $(4 \times)^{167}$, w3.t ntr „Weg des Gottes“ $(4 \times)^{168}$, w3.t Hrw „Weg des Horus“ $(4 \times)^{169}$, w3.t Dhwtj „Weg des Thot“ $(4 \times)^{170}$, w3.t jmn.t „Weg des Westens“ $(3 \times)^{171}$, w3.t $R^{c}$,Weg des Re“ $(3 \times)^{172}$, w3.t 3h(.w) ,Weg des Totengeistes/der Totengeister“ $(2 \times)^{173}$, w3.t Nw.t „Weg der Nut“ $(2 \times)^{174}, w 3 . t j 3 d w$,Weg der Seuche“ $(1 \times)^{175}$.

Dies ändert sich im Prinzip auch dann nicht, wenn das Rectum erweitert ist: $w 3 . t R$-st $3 . w$ „Weg von Ra-Setjau“ $(4 \times)^{176}$, w3.t 3w.t jb ,Weg der Freude“ $(3 \times)^{177}$, w3.t nb tm „Weg des Allherrn“ $(2 \times)^{178}$, w3.t wj3 Hprj „Weg der Barke des Chepri“ (1× $)^{179}$, w3.t Jnpw nb qrs.t „Weg des Anubis, Herrn des Begräbnisses“ $(1 \times)^{180}$. Keine nota genitivi steht auch in w3.t NN tn „Weg dieser NN“ $(1 \times)^{181}$, das aus dem w. $t=j$ der Parallele umgesetzt wurde. Für dieses Wort stehen zwei Belege mit erweitertem Regens zur Verfügung. Sie zeigen, wie zu erwarten, die nota genitivi: $w 3 . t$ nb.t n.t $s \underline{d} . t$, ,jeder Weg des Feuers“ $(1 \times)^{182}$, w3.t tw n.t nb.w k3.w „dieser Weg der Herren der Kas“ (1× ${ }^{183}$.

Es gibt jedoch noch mehrere weitere Belege mit nota genitivi. Bei den folgenden steht, ähnlich wie wir es schon oben gesehen haben, als Rectum ein substantiviertes Adjektiv: w3.t n.t ${ }^{c}$ 3.t ,Weg der Großen“ w3.t n.t ${ }^{c} 3$ ntr.w „Weg des Großen der Götter“ (je $1 \times)^{184}$, w3.t n.t dpj.w t3 „Weg

166 CT VII 317c, VII 513e. Die prinzipiell ebenfalls denkbare Interpretation von $M^{3}{ }^{c} . t$ als Adjektiv ist hier unwahrscheinlich, weil zwei Textzeugen den Klassifikator der Göttin verwenden.

167 CT II 20d, IV 81a.

168 CT III 345a.

169 CT II 231c.

170 CT VII 371j.

171 CT I 89c, IV $366 \mathrm{~g}$.

172 CT VII $414 \mathrm{~b}$.

173 CT V 329b.

174 CT II 20 d.

175 CT VII 412c.

176 CT VII 339e, VII 521a.

177 CT IV 171b.

178 CT I 211a.

179 CT V $290 \mathrm{~m}$.

180 CT VI $94 \mathrm{~h}$.

181 CT IV 171b.

182 CT VI 388b.

183 CT VI 198u.

184 CT VI 192e. Ich gehe davon aus, dass diese Ausdrücke grammatisch wohlgeformt sind, auch wenn die Textüberlieferung in beiden Textzeugen verderbt zu sein scheint. 
der Erdbewohner“ $(1 \times)^{185}$. Der indirekte Genitiv ist unter dieser Bedingung jedoch nicht obligatorisch, wie der Ausdruck w3.t ntr. (j)t „Weg des Göttlichen (sc. Auges)“ (4× $)^{186}$ zeigt. Ich kann nur spekulieren, dass jeweils der Grad der lautlichen Eindeutigkeit in der gesprochenen Sprache bestimmt haben mag, ob die nota genitivi erforderlich erschien oder nicht.

Die Stelle CT VII $305 f$ hat w3.t sd.tjw $(2 \times) \sim$ w 3.t n.t $s \underline{d} . t j w(1 \times)$,Weg der Feurigen“, wo ich das $n$.t der Variante auch wieder durch das substantivierte Adjektiv erklären würde. In CT VII 504a steht nun w3.t n.t sd.t (1×) ,Weg des Feuers“. Obwohl de Buck aus CT VII 504a-c einen separaten Spruch Nr. 1157 macht, handelt es sich eigentlich nur um eine Parallele zu CT VII 305f-h. Daher kann w3.t n.t s d.t aus $w$ 3.t n.t s. d.tjw verderbt und das $n$.t wieder auf diese Weise zu erklären sein.

So bleibt ein einziger Beleg mit einem indirekten Genitiv übrig, für den keine Motivation erkennbar ist: $w 3 . t$ $n . t R$-st $\underline{3} . w$,Weg von Ra-Setjau“ ( $(1 \times)^{187}$ (neben häufigerem direktem Genitiv, siehe oben). Die Stellen $w n . n=j / N N$ w $3 . t$ $n R^{c}(2 \times)^{188}$ und sšm $=j$ w3.t $n R^{C}(1 \times)^{189}$ können wegen der mangelnden Kongruenz sicher nicht als indirekter Genitiv gelesen werden, sondern müssen dativisch verstanden worden sein („ich öffnete/zeige den Weg dem Re“), auch wenn dieses Textverständnis vermutlich nicht das originale ist.

Die bis hierher behandelten vier Substantive $z 3$ „Sohn“, $p r$ „Haus“, $n b$ „Herr“ und w3.t „Weg“, denen man sicherlich viele weitere an die Seite stellen könnte, verhalten sich, wie ich vermute, hinsichtlich der Genitivkonstruktion alle identisch und konstituieren somit eine Klasse. Diese Klasse hat die folgenden Eigenschaften: (1) Auf diese Substantive folgt das Rectum als direkter Genitiv. (2) Dies gilt uneingeschränkt auch dann, wenn das Rectum erweitert ist. (3) Wenn aber das Regens durch ein Attribut erweitert ist, so muss dieses entweder der direkten Genitivgruppe nachfolgen (eher selten, da potentiell ambig), oder (die häufigere Variante) es wird der indirekte Genitiv gewählt und das Attribut kann dann direkt auf sein Bezugswort folgen. (4) Außerhalb dieser Bedingung tritt der indirekte Genitiv normalerweise nur dann ein, wenn ohne ihn die Gefahr einer Ambiguität bestünde. Dies kann insbesondere der Fall sein, wenn das Rectum aus einem substantivierten Adjektiv besteht, doch ist auch dann der indirekte Genitiv nicht obligatorisch. Hier mag der Grad

185 CT III $317 \mathrm{~g}$.

186 CT VII 497a, VII $497 \mathrm{k}$.

187 CT VII 521a.

188 CT V $337 \mathrm{~b}$.

189 CT VII 414b. der Eindeutigkeit in der gesprochenen Sprache eine Rolle gespielt haben, was aber für uns schwer nachvollziehbar ist. (5) Schließlich sind ganz vereinzelte weitere Fälle von indirekten Genitiven zu verzeichnen, die derzeit nicht erklärt werden können.

Nun möchte ich vier Substantive einer zweiten Klasse behandeln, die sich praktisch entgegengesetzt verhält, indem sie den indirekten Genitiv bevorzugt. Ich beginne mit:

\section{$j b$ „Herz“}

Dieses Substantiv erfordert nach sich in der Regel den indirekten Genitiv, und zwar auch schon dann, wenn keines der beiden Nomina expandiert ist: $j b n W_{s j r}$ „Herz des Osiris“ $(19 \times)^{190}, j b$ n ntrr.w „Herz der Götter“ $(13 \times)^{191}, j b$ $n z$ „Herz eines Mannes“ $(11 \times)^{192}, j b n$ 3s.t „Herz der Isis“ $(9 \times)^{193}, j b n R^{c}$ „Herz des Re“ $(9 \times)^{194}, j b n$ ih.w „Herz der Totengeister“ $(7 \times)^{195}, j b$ n Jnpw „Herz des Anubis“ $(7 x)^{196}$, $j b n \underline{d} \underline{j}$ d $3 . t(=f)$ „Herz des/seines Tribunals“ $(5 \times)^{197}, j b n$ $b 3=k$,Herz deines Ba“ $(3 \times)^{198}, j b n k 3 . w(=s n)$ „Herz der/ ihrer Stiere“ $(3 \times)^{199}, j b n$ N.t „Herz der Neith“ $(2 \times)^{200}, j b n$ $n \underline{t r}$ „Herz des Gottes“ $(2 \times)^{201}, j b$ n hm.t „Herz einer Frau“ $(2 \times)^{202}, j b n$ sbk.w „Herz der Krokodilgötter“ $(2 \times)^{203}, j b n$ $w r$ „Herz des Großen“ $(1 \times)^{204}, j b n$ mw. $t=f$ „Herz seiner Mutter“ $(1 \times)^{205}, j b$ n rmt.w „Herz der Menschen“ $(1 \times)^{206}, j b$ $n$ sn. $t j=k(j)$ „Herz deiner beiden Schwestern“ $(1 \times)^{207}, j b n$ Sth „Herz des Seth“ (1× $)^{208}, j b n$ Gb „Herz des Geb“ $(1 \times)^{209}$.

190 CT II 246a, VI 266i, VII 385b.

191 CT II 245d, III 157c, VI 45f, VI 316f.

192 CT II 126d, II 129h, V 45a, V 54a, V 332h, VI 164j, VI 227a, VI 344l, VI 366 a.

193 CT I 23b, IV 22c.

194 CT I 76g, I 202e, II $245 \mathrm{~d}$.

195 CT II 245d, V 297g, V 307b.

196 CT I 198f.

197 CT I 76h.

198 CT I $182 f$.

199 CT IV $155 \mathrm{~g}$.

200 CT VI 170k.

201 CT VI 227g.

202 CT VI 191m.

203 CT VI 170j.

204 CT VI $361 \mathrm{~b}$.

205 CT I 80m.

206 CT V 333q.

207 CT VI 325q.

208 CT IV 85q.

209 CT IV 860. 
Daran ändert sich nichts, wenn das Rectum erweitert wird. Da schon ein einfaches Rectum den indirekten Genitiv erfordert, wird man denselben hier erst recht erwarten, weshalb ich von solchen Fällen nur eine Auswahl zitiere ohne Anspruch auf Vollständigkeit: $j b n h n t j z h$ $n t r$ „Herz des Vorsitzenden der Gotteshalle“ $(11 \times)^{210}, j b n$ $k 3(. w) h r j(. w)$ psd.t „Herz des Stieres/der Stiere, der/die vor der Neunheit ist/sind“ $(6 \times)^{\mathbf{2 1 1}}$, jb n jmj.w šmsw „Herz derer, die im Gefolge sind“ $(6 \times)^{212}, j b$ n jmj.w b 3 h „Herz derer, die vorne sind“ $(4 \times)^{213}, j b$ n jrr $r=k$,Herz dessen, der gegen dich handelt“ $(4 \times)^{214}, j b n z p n / p f$ „Herz dieses/ jenes Mannes“ $(4 \times)^{215}, j b n$ St $\underline{h} h f t j=f$,Herz seines Feindes Seth“ $(4 \times)^{216}, j b n$ (mwt) mwt.t „Herz (eines Toten und) einer Toten“ $(3 \times)^{\mathbf{2 1 7}}, j b$ n Hrw nd $j t j=f$, Herz des Horus, Rächers seines Vaters“ $(3 \times)^{218}, j b n$ ps d.t.t(j) c3.t(j) „Herz der (beiden) großen Neunheit(en)“ $(2 \times)^{219}, j b n z$ z $=s$ Hrw „Herz ihres Sohnes Horus“ $(2 \times)^{220}$. Auch bei erweitertem Regens (etwa *,dieses Herz des X“) würde man einen indirekten Genitiv erwarten, doch kann ich diese Situation mit $j b$ nicht belegen.

Wenn manche Textzeugen in einem $j b=j / f / s$ des Urtextes das Pronomen durch den Personennamen ersetzen, so entsteht meist $j b n N N$ pn/tn „Herz dieses/dieser NN“ mit indirektem Genitiv $(25 \times)^{221}$. Viel seltener, aber doch auch, ist ein $j b N N$ pn/tn mit direktem Genitiv zu belegen $(3 \times)^{222}$. Da die Ersetzung des Pronomens durch den Namen oft recht mechanisch erfolgte, ist die Annahme vertretbar, dass der Schreiber nur vergaß, das grammatisch erforderliche $n$ einzufügen. Umgekehrt ist die Tatsache, dass überhaupt meistens ein $n$ hinzugefügt wurde, ein starkes Argument dafür, dass man den indirekten Genitiv als erforderlich oder zumindest als grammatisch besser empfand.

Der direkte Genitiv ist nach $j b$ selten, aber doch belegt, und zwar in den folgenden fünf Verbindungen, von denen die ersten vier - wie oben gezeigt - auch mit indirektem Genitiv vorkommen: $j b$ Stš „Herz des Seth“ $(5 \times)^{223}, j b 3 s . t$

210 CT I 198g, I 220h.

211 CT V 377b, V 386c.

212 CT I 239c, IV $124 \mathrm{f}$.

213 CT I 220c.

214 CT I 187a.

215 CT II 95g, II 103b.

216 CT V 322l, V 325g.

217 CT II 93 f.

218 CT I 223d.

219 CT IV 30j.

220 CT I 80n.

221 CT I 23a, II 184a, II 194b, IV 2a, V 296b, V 346a, V 367e, V 368f, V 376i, V 383k, VI 355k.

222 CT I 23a, III 157b, V 58b.

223 CT VII 58e, VII 61w, VII 62bb, VII 62cc.
„Herz der Isis“ $(2 \times)^{224}, j b R^{c}$ „Herz des Re“ $(2 \times)^{225}$, jb jmj.w $b 3 h$ „Herz derer, die vorne sind“ $(1 \times)^{226}, j b$ ntr.w $P$,Herz der Götter von Pe“ $(1 \times)^{227}$. Der Ausdruck $j b$ Stš scheint auffällig häufig zu sein, jedoch betreffen alle Belege die Phrase jnildi pd.t jb Stš „bringen/geben, was das Herz des Seth erfreut", die pyramidentextlichen Ursprungs ist (CT Spruch 855 = PT Suppl. 57E-57 J = Faulkner 1969, 1f.) und mit der seltenen Verbindung $p \underline{d} j b$ auch lexikalisch auffällt. So würde ich diese und möglicherweise auch die anderen Belege des direkten Genitivs nach $j b$ als Reste älteren Sprachgebrauchs bewerten. Den obskuren Ausdruck hw jb tzm, der mehrfach in CT II 166-174 vorkommt (Hannig 2006, 1624), lasse ich unberücksichtigt.

Ich will noch kurz auf den Plural $j b . w$ zu sprechen kommen, der als Regens einer Genitivverbindung in den Sargtexten leider nur in einer einzigen Phrase belegt ist. Diese zeigt bemerkenswerterweise den direkten Genitiv: jb.w 3h.w „Herzen der Totengeister“ $(3 \times)^{228}$. Wenn wir diesen Ausdruck belasten wollen, so scheint $j b$ auch in den Sargtexten in eine Reihe zu gehören mit „den einsilbigen Maskulina [...], die in den Pyramidentexten im Singular das Rectum im indirekten Genetiv anschließen, im Dual und Plural aber im direkten." (Jansen-Winkeln 2000, 33 auf der Basis der Daten aus Sander-Hansen 1936).

Gesondert behandeln möchte ich die Ausdrücke der Form jm.j jb (n) X ,der im Herzen des X ist = Liebling des $X^{\prime \prime}$. Bei diesen ist zweifelhaft, ob die syntaktische Klammerung als [jm.j jb] $n X$ oder als jm.j [jb $n X]$ anzusetzen ist; nur in letzterem Fall würden sie überhaupt hierher gehören. Die wenigen Belege sind nicht eindeutig und zeigen sowohl den indirekten Genitiv: jm.j jb $n$ nb ntr.w „der im Herzen des Herrn der Götter ist = Liebling des Herrn der Götter“ $(2 \times)^{229}, j m . j$ jb $n n b k 3 r$, der im Herzen des Herrn des Schreins ist“ $(1 \times)^{230}$, als auch den direkten: jm.j jb $n b$ $3 h . t$,der im Herzen des Herrn des Horizontes ist“ $(2 \times)^{231}$. Das einzige verfügbare Beispiel mit pluralischem $j b . w$ hat einen direkten Genitiv: $j m[. j]$ jb.w hrj. $w$ - $d p$, der in den Herzen der Vorsteher ist“ $(1 \times)^{232}$.

Auf der Basis von $j b$ „Herz“ bildet das Ägyptische die Komplexpräposition $h r$-jb $X$ „auf dem Herzen von X $=$ inmitten von $X^{“}$ sowie eine dazugehörige Nisbe $h r . j-j b$ $X$,inmitten von $X$ befindlich“, beide in der Graphie nicht
224 CT I 23b.
225 CT I 202e.
226 CT I 220c.
227 CT 12440.
228 CT V $297 \mathrm{~g}$, V $315 \mathrm{~h}$.
229 CT IV 144g, IV $145 \mathrm{e}$.
230 CT VI $267 \mathrm{t}$.
231 CT IV $114 \mathrm{f}$.
232 CT VI 268s. 
immer klar voneinander zu trennen. In diesen häufigen Ausdrücken, die ich hier nur anhand weniger Beispiele vorstelle, steht in aller Regel der direkte Genitiv: $h r$-jb p.t „inmitten des Himmels“ $(24 \times)^{233}, h r-j b \underline{t} 3-w j=f j$,inmitten seiner beiden Küken“ $(20 \times)^{234}, h r(. j)$-jb jr.t=j/k/f, inmitten meines/deines/seines Auges (befindlich)“ (19x ${ }^{235}, h r$-jb $w t n w$,inmitten der Flut“ (13× $)^{236}, h r . j-j b d b n=f$, in seiner Umkreisung befindlich“ $(13 \times)^{237}, m$ hr-jb $n f r=f$,inmitten seiner Schönheit“ $(12 \times)^{238}, h r(. j)-j b$ pr $=f$, inmitten seines Hauses (befindlich)“ (9x ${ }^{239}, h r$-jb $3 h$.t , ,inmitten des Horizonts“ $(8 \times)^{240}, h r-j b k k w$,inmitten der Dunkelheit“ $(8 \times)^{241}$, $h r$-jb sh.t j3rw ,inmitten des Binsengefildes“ $(3 \times)^{242}, h r . j-$ $j b$ hw.t Sth „im Tempel des Seth befindlich“ $(2 \times)^{243}, h r-j b$ db3.t n.t $w^{c}$ b.w ,inmitten des Ankleideraumes der Reinen“ $(2 \times)^{244}$. Die Verwendung des direkten Genitivs zeigt uns, dass dieser Ausdruck sich schon in einem fortgeschrittenen Stadium der Grammatikalisierung befand und nicht mehr wortwörtlich als „im Herzen von ...“ verstanden wurde. Synchron ist wohl gar nicht mehr von einem „Genitiv“, sondern von einem Präpositionalkomplement zu sprechen. Der grammatikalisierte Charakter wird auch dadurch bestätigt, dass die Kongruenzmerkmale der Nisbe meist an das Ende der Verbindung $h r . j-j b$ angehängt werden (Wb III 137), wie in $h r . j-j b . t ~ d s ̌ r . w=s$,die in ihrem Zorn befindliche“ $(24 \times)^{245}, h r . j-j b . t$ Jwnw ,die in Heliopolis befindliche“ $(5 \times)^{246}, h r . j-j b . w j w$ nsrsr ,die inmitten der Flammeninsel befindlichen“ $(1 \times)^{247}$.

Einzelne Fälle mit $n$ lassen wohl immer eine spezielle Erklärung zu. In CT II 364a schreiben die meisten Textvertreter $s b^{3}$ pw hr(.j)-jb „dieses mittlere Tor“. Zwei Textzeugen erweitern dies zu $s b^{3} p w h r(. j)$-jb $n$ p.t, was also vermutlich nicht etwa bedeutet * ,dieses Tor inmitten des Himmels“, sondern „[dieses mittlere Tor] des Himmels", womit das $n$ regulär wegen des komplexen Regens steht. Ganz entsprechend ist $j w N N$ rh $s b^{3} p w h r(. j)-j b$

233 CT III 369b, III 371b, III 394b, V 21e, V 214c, VI 166l, VI 251f, VII 19j, VII 472d.

234 CT IV 276a, IV 276c.

235 CT V 378b, V 385k, VI 224f, VI 262b, VII 16v, VII 118d, VII 177k, VII 222h, VII 409b.

236 CT V 215a, V 216c, V 220b, V 225l, V 226a, V 226 h.

237 СТ I $346 \mathrm{c}$.

238 CT I 356b.

239 CT IV 370b.

240 CT III 372a, III 379c, III 394d.

241 CT V 216b, V 216c, VI $200 \mathrm{e}$.

242 CT III 380c.

243 CT VI $252 \mathrm{e}$.

244 CT I $170 \mathrm{~h}$.

245 CT I 378c, I 382b.

246 CT V 21d, VII 2421.

247 CT VI $295 f$. $n$ sh.t-j3rw in CT II 388i (1x) wohl nicht zu verstehen als „N connaît cette porte [qui se trouve au milieu du Champ des Roseaux]“ (Carrier 2004, 399, Klammern von mir), sondern als „N connaît [cette porte centrale] du Champ des Souchets“ (Barguet 1986, 577, Klammern von mir). Kein Grund für den indirekten Genitiv ist mir ersichtlich in dem $6 \times$ belegten $h r . j$-jb.w n.w $t 3$, ?die inmitten der Erde befindlichen" gegenüber einmaligem $h r . j-j b . w t 3$ in CT I $60 \mathrm{c}$, jedoch scheint der Passus als ganzer korrupt zu sein.

\section{$r n, N^{N a m e}{ }^{6}$}

In dieselbe Klasse gehört das Substantiv $r n$ „Name“. Einen Großteil der Belege liefern die Fährmannsprüche, in welchen die Namen der einzelnen Schiffsteile behandelt werden. Im Regelfall steht nach $r n$ der indirekte Genitiv: $r n$ $n$ h $3 t . t=f / s$,Name seines/ihres Vordertaues“ $(12 \times)^{248}, r n n$ $R$-st 3 .w „Name von Ra-Setjau“ (10× $)^{249}$, $r n n R^{c}$,Name des $\operatorname{Re}^{\prime \prime}(8 \times)^{250}, r n n h m w / y(=s)$ „Name des/ihres Ruder(er)s“ $(8 \times)^{251}, r n n w^{c}$ „Name des einen“ $(6 \times)^{252}, r n n m h n . t j(=s)$ „Name des/ihres Fährmannes“ $(5 \times)^{253}, r n n m$ m $3 . t(=f / s)$ „Name des/seines/ihres Stockes(?)“ (5× $)^{254}$, rn $n \underline{d n h}$. $w j=k / f$,Name deiner/seiner Ruderblätter“ $(5 \times)^{255}, r n n$ shn. $t=s$ „Name ihres Knüpfwerks“ $(4 \times)^{256}, r n n$ st 3 . $. t=s$ „Name ihres Treideltaus“ $(4 \times)^{257}, r n n$ i h. $t=s$ „Name ihres Feldes“ $(3 \times)^{258}, r n n w \underline{d} . w t(=s)$,Name der/ihrer Pflöcke(?)“ (3x $)^{259}$, $r n n$ hn. $t=f$,Name seiner Fahrt“ $(3 \times)^{260}, r n$ n sw. $t=s$,Name ihrer Binse“ $(3 x)^{261}$, rn $n$ jmn.t „Name des Westens“ $(2 \times)^{262}$, $r n n$ jrj. $w=s n$,Name der zu ihnen gehörigen“ $(2 \times)^{263}, r n n$ Jtmw „Name des Atum“ $(2 \times)^{264}, r n n$ jtrw(.w) „Name des Flusses/der Flüsse“ $(2 \times)^{265}, r n n m h^{c}=s$,Name ihres Flach-

248 CT V 71f, V 71g, V 204c, VI $12 f$.

249 CT VII 358c.

250 CT VII 399a.

251 CT V 71c, V 71h, V 194e, V 206h, VI 11b, VII $10 d$.

252 CT IV 346i, IV 346j.

253 CT V 70h, V 71a, V 71b.

254 CT VI 6h, VI 18c.

255 CT V 193e, V 206j.

256 CT VI 5 d.

257 CT VI 5 f.

258 CT VI $4 \mathrm{i}$.

259 CT V 189d, V $204 i$.

260 CT V 71b.

261 CT VI 12d.

262 CT VII 9f, VII 10g.

263 CT VII 473k.

264 CT V 318h.

265 CT I 307e, V 207b. 
ses“ $(2 \times)^{266}, r n n n f w$ „Name des Schiffers“ $(2 \times)^{267}, r n n$ $h r p w(=s)$ „Name des/ihres Schlegels“ $(2 \times)^{268}, r n n z 3 w=f$ „Name seines Balkens“ $(2 \times)^{269}, r n n \operatorname{sm}^{c} . w=s$,Name ihrer Stangen“ $(2 \times)^{270}, r n n \check{s}^{c} . t=f / s$,Name seines/ihres Messers“ $(2 \times)^{271}, r n n$ šnw $(=s)$,Name des/ihres Netzes(?)“ $(2 \times)^{272}, r n$ $n \underline{t} \underline{3} w$,Name des Windes“ $(2 \times)^{273}, r n n d b h . w=s$,Name ihrer Manövriertaue(?)“ $(2 \times)^{274}$, dazu kommen weitere Streubelege $(19 \times)^{275}$.

Einige Male ist auch der direkte Genitiv belegt, jedoch immer nur als minoritäre Lesart in Phrasen, die auch mit indirektem Genitiv vorkommen: $r n n$ wsr.w $(=f / s)$,Name der/seiner/ihrer Ruder“ $(10 \times)^{276} \sim r n$ wsr.w $(1 \times)^{277}, r n n$ ${ }^{c} \underline{d}(=f / . w=s)$,Name der Spule/seiner Spule/ihrer Spulen“ $(5 \times)^{278} \sim r n^{c} \underline{d}(1 \times)^{279}, r n n m h s f$,Name des Spannpflockes“ $(3 \times)^{280} \sim r n m h s f(1 \times)^{281}, r n n m \underline{d} 3 b . t=s$,Name ihrer Schöpfkelle“ $(2 \times)^{282} \sim r n m d \underline{d} b . t=s(1 \times)^{283}, r n n$ h $h^{c} . t$,Name der Masthalterung(?)“ $(1 \times)^{284} \sim r n h^{c} . t(1 \times)^{285}$. Auch wenn der Grund für die Verwendung des direkten Genitivs unklar bleibt, handelt es sich bei ihm jedenfalls eindeutig um die weniger übliche Konstruktion.

Wenn das Rectum erweitert ist, steht der indirekte Genitiv ebenfalls fast obligatorisch. Für diese Situation genügt eine Auswahl von Belegen ohne Anspruch auf Vollständigkeit: $r n \quad n$ hf $3 . w \quad p n / p f$ „Name dieser/jener Schlange“ (10x) $)^{286}, r n n$ 3h 7 (jpw) „Name d(ies)er sieben Totengeister“ $(7 \times)^{287}, r n n n$ '.wt sp3.wt (r.w) hn.w/jmj.w sh.t htp „Name der Städte, Gaue (und Seen) inmitten des Opfergefildes“ $(6 \times)^{288}, r n n$ dp.t $t n(r-d r=s)$ „Name dieses

266 CT VI $5 b$.

267 CT V 194a, V 194c.

268 CT V 189b, V 204g.

269 CT V $193 f$.

270 CT VI $11 \mathrm{~h}$.

271 CT VI 6d, VI $18 \mathrm{e}$.

272 CT VI 7b.

273 CT V 194g, V 207g.

274 CT V $190 \mathrm{~g}$.

275 CT V 71b, V 190c, V 204e, V 205b, V 206a, V 206f, V 207k, V 318k, VI 4g, VI 18g, VI 22k, VI 22m, VI 35h, VI 44m, VI 142f, VI 291n, VI $307 \mathrm{t}$, VII 37o, VII $137 i$.

276 CT V 71d, V 71e, V 191c, VI 11f.

277 CT V 191c.

278 CT VI 6b, VI 11h, VI 17n, VI 22a.

279 CT VI 6b.

280 CT VI 6f, VI 18 a.

281 CT VI $6 f$.

282 CT V 204m, VI 12a.

283 CT V 72a.

284 CT V $189 f$.

285 CT V $189 f$.

286 CT II 378c.

287 CT IV 262b.

288 CT V 364e, V $381 n$. (ganzen) Schiffes“ $(5 \times)^{289}, r n n w^{3}{ }^{3} p n$ „Name dieser Barke“ $(4 \times)^{290}, r n n n \underline{t} r(=t n) p w \underline{d} \underline{d} . w(=\underline{t} n) n=f \underline{d} f 3 . w r$ fn $\underline{d}=f$ „Name dieses (eures) Gottes, dem ihr/man Nahrung an seine Nase gebt/gibt“ (4×) ${ }^{291}, r n n z p w h 33$ ( $\left.r=s\right)$ „Name dieses Mannes, der (zu ihr) hinabsteigt“ (4× $)^{292}, r n n w h^{c} . w$ h3m.w-s(j) „Name der Fischer, die es (das Netz) werfen“ $(3 \times)^{293}$, rn $n$ 3h.w z 33.w w3.wt jptn „Name der Totengeister, die diese Wege bewachen “ $(2 \times)^{294}$. Nur zweimal ist als isolierte Textvariante ein direkter Genitiv belegbar: $r n n n t r$ pw ${ }^{c} n t j$ jm $=s$ „Name dieses großen Gottes, der in ihr ist“ (15×) rn ntr pw ${ }^{c} 3$ ntj jm $=s(1 \times)^{295}, r n n{ }^{c} m^{c} 3 . t=k t w$ qm 3 . $y t h 3=k$ „Name dieses deines Wurfholzes, das dir hinterher geworfen wird“ (8× $\sim r^{c}{ }^{c}{ }^{c} 3 . t=k \ldots(1 \times)^{296}$. Wenn das Regens erweitert wird, was bei $r n$ vergleichsweise gut belegt ist, steht immer der indirekte Genitiv. Ich zitiere wieder nur eine Auswahl von Stellen: $r n=k n Q b h$-sn. $w=f$, dein Name (des) Qebehsenuef“" $(9 \times)^{297}, r n=k n \quad D w 3-m w . t=f$ „dein Name Duamutef“ $(8 \times)^{298}, r n=k n$ Hrw „dein Name Horus“ $(2 \times)^{299}, r n=k$ pw $n$ Hpy „dieser dein Name Hapi“ $(5 \times)^{300}, r n=j p w n$ Hw.t-Hrw ,dieser mein Name Hathor“ $(4 \times)^{301}$.

$X p w r n(n) \check{s} m 3^{c}(\text { je } 1 \times \text { mit und } 1 \times \text { ohne } n)^{302}$ dürfte am ehesten bedeuten „X ist der wahre Name des Sees“ (mit Barguet 1986, 632), obwohl beide Varianten grammatisch schwierig sind: der direkte Genitiv ist nach $r n$ abnorm, und im indirekten Genitiv sollte sich das Adjektiv nicht auf das Regens beziehen können. Auch eine Interpretation von $m 3^{3}$ als Adverb wäre denkbar: „... ist wahrhaft der Name des Sees“. Zwei weitere Textzeugen schreiben wohl verderbt $r n \check{s} m^{3 c}$ mit $r$,Mund“, was bestätigt, dass die Stelle Schwierigkeiten bereitete. Darauf aufbauende Übersetzungen wie „Der Eingang des wahrhaften Sees“ (Backes 2005, 71) halte ich für irrig.

Wo einzelne Textzeugen aus einem Pronomen den Personennamen hergestellt haben, wurde entweder (korrekter) das $n$ eingefügt: $r n n N N$ pn/tn „Name dieses/r

289 CT V 193h, V 203l, V 204b, V 206k.

290 CT VII 454b.

291 CT V 197b.

292 CT VI 7g, VI 13a.

293 CT VI 18k, VI 220, VI $24 \mathrm{~g}$.

294 CT VII 499c.

295 CT IV 196a.

296 CT VII 425b.

297 CT VI 115a, VI 116b. Hier und in ähnlichen Fällen gebraucht das Ägyptische einen Genitiv, der im Deutschen nicht idiomatisch wäre.

298 CT VI 113e, VI 113i.

299 CT VI 116h.

300 CT VI 112b.

301 CT IV 175e.

302 CT VII $306 g$. 
NN“ $(5 x)^{303}$, oder die Einfügung konnte (wohl aus Nachlässigkeit) unterbleiben: $r n N N$ pn/tn $(4 \times)^{304}$. An den beiden Belegstellen, wo das Regens noch durch ein Attribut erweitert ist, steht erwartungsgemäß das $n: r n$ pn $n N N$ $p n$,dieser Name dieses NN“ $(1 \times)^{305}, r n$ fr $n$ NN pn , der schöne Name dieses NN“ $(1 \times)^{306}$.

Bemerkenswert ist, dass der Plural desselben Nomens mit direktem Genitiv konstruiert werden kann. Und zwar scheint dies den Regelfall darzustellen, wenn auch die schwache Belegzahl für statistische Aussagen kaum genügt: $r n . w$ jtrw.w „Namen der Flüsse“ $(3 \times)^{307}, r n . w$ šm.w „Namen der Gehenden“ (1x) ${ }^{308}$, (indirekt:) rn.w n.w q33 pf „Namen jener Windung“ $(1 \times)^{309}$. Erwartungsgemäß finden wir den indirekten Genitiv, wenn das Erstnomen erweitert ist: $r n . w=\underline{t} n$ jpw n.w jr.t Hrw ,diese eure Namen des Horusauges“ $(2 \times)^{310}$.

\section{$k 3$, „Ka“}

Als drittes Substantiv behandle ich $k^{3}$, Ka“, das, wie ich annehme, ebenfalls in die Gruppe mit regelmäßigem indirektem Genitiv gehört. Doch sind bei diesem Wort die Belege schon viel geringer an Zahl und die Schlussfolgerungen daher weniger sicher. Wir finden also nach $k 3$ den indirekten Genitiv: $k 3, n z$,Ka eines Mannes“ $(3 \times)^{311}, k^{3} n$ $R^{c}$,Ka des Re“ $(2 \times)^{312}$. Die Phrase $k 3 n m w . t=f$, Ka seiner Mutter“ $(4 \times)^{313}$ hat allerdings auch eine etwas seltenere Variante $k 3 m w . t=f(2 \times)^{314}$ mit direktem Genitiv. Der indirekte Genitiv erscheint auch bei komplexem Rectum: $k 3 n$ $n$ tr $n b$ „Ka jedes Gottes“ $(2 \times)^{315}, k 3 n$ ntr.w nb. $w$ „Ka aller Götter“ $(1 \times)^{316}$; Belege mit komplexem Regens fehlen.

Die geläufige Phrase $n k 3 n N N$,für den Ka des/der NN“, die in der ägyptischen Opferformel abertausende Male belegt ist - und zwar, wie man sich erinnern wird, in aller Regel mit $n-$, kommt auch in den Sargtexten in ih-

303 CT II 162i, IV 390c, VI 312d, VI 368h, VII 460b.

304 CT II 400a, V 51c, V 318g, VI $397 f$.

305 CT V $236 \mathrm{f}$.

306 CT VI 402e.

307 CT I 307 e.

308 CT VI 236n.

309 CT IV 1471 .

310 CT III $339 f$.

311 CT III 359d.

312 CT III 394k, VI 391n.

313 CT II 60c.

314 CT II 60c, VI 403q.

315 CT VI 392j, VII 111s.

316 CT VI 392m. rer regulären Form vor $(4 \times)^{317}$. Einmal jedoch ${ }^{318}$ erscheint sie mit direktem Genitiv als $n k 3 N N$, was als abnorm einzuschätzen ist. Dort, wo einige Textzeugen für ein $k j=j / f$ der Textvorlage den Personennamen eingesetzt haben, erscheint ebenfalls weit überwiegend der indirekte $\mathrm{Ge}$ nitiv: $k 3 n N N(p n / t n)$ „Ka d(ies)es/d(ies)er NN“ (11× $)^{319}$; gerade hierin sehe ich einen starken Hinweis darauf, dass nach $k 3$ tatsächlich der indirekte Genitiv regulär war. Dem steht ein einziger Beleg für $k 3 N N$ tn „Ka dieser NN“ gegenüber ${ }^{320}$.

Bemerkenswert an diesem Substantiv ist wie schon bei den zuvor behandelten, dass es im Plural den direkten Genitiv erfordert, wie alle verfügbaren Belege zeigen: $k 3 . w$ $3 h . w$ „Kas der Totengeister“ $(14 \times)^{321}, k 3 . w N N$ pn „Kas dieses NN“ $(2 \times)^{322}, k$ 3.w ntr.w „Kas der Götter“ (1× $)^{323}$.

\section{$n w$, dies $^{66}$}

Die neutrischen Demonstrativa $n w, n n$ und $n 3$ folgen, im Gegensatz zu den meisten Demonstrativa, nicht wie ein Adjektiv ihrem Bezugswort, sondern sind syntaktisch einem Substantiv äquivalent: Sie stehen am Anfang der Nominalphrase und können etwa ein Adjektiv nach sich haben (z. B. $n w ~ m r$ zp-2 „dieses sehr schmerzhafte“, CT V 277g) oder aber einen Genitiv, der dann regelmäßig indirekt konstruiert wird (Gardiner 1957, §111; vgl. weiter die zwei verfügbaren Spezialuntersuchungen $\mathrm{zu} n w$ in den Sargtexten von el Toukhy 1993 und Vernus 1994). Die Verbindung Demonstrativum- $n$-X (wörtlich „dies von X“) entspricht semantisch meist einem pluralischen „diese X“, besonders im Sprachgebrauch des Alten Reiches (Edel 1955/64, 89) manchmal aber auch einem singularischen „diese(r/s) X“, was jedoch auf die Konstruktion des Genitivs keinen Einfluss hat. In den Sargtexten überwiegt die pluralische Lesart, so dass diese Demonstrativa dann in Konkurrenz zum (adjektivisch konstruierten) jpw/jptw „diese“ stehen.

Insofern die neutrischen Demonstrativa gewöhnlich den indirekten Genitiv verlangen, gehören sie in dieselbe Klasse wie die vorgenannten Substantive. Ich behandle hier nur $n w$, das häufigste von ihnen, und übergehe $n n$,

317 CT I 40d, I 313.T2C, VI 123a, VII 47a.

318 CT VII 104 f.

319 CT I 166h, III 9b, IV 5d, VI 26d, VI 110d, VI 293h, VI 350a, VII 413a.

320 CT III 9b.

321 CT V 342b, V 366d, V 382g.

322 CT V 295d, V 372e.

323 CT II $52 i$. 
das aufgrund der Komplementierung mit keinem, einem oder zwei $n$-Zeichen erhebliche graphische Unsicherheiten bereitet, sowie das in den Sargtexten noch seltene $n$ 3.

Zunächst fällt auf, dass eine Verbindung ohne weitere Attribute, etwa ein * $n w n$ ntr.w ,diese Götter“, in den Sargtexten kaum vorkommt. Wie es scheint, hat man in diesem Fall das adjektivische Demonstrativum bevorzugt wie z. B. in ntr.w jpw „diese Götter“ $(6 \times)^{324}$. Als unerweiterte Kandidaten finde ich in den Sargtexten zwei schwierige Stellen, in denen noch die ursprüngliche Bedeutung „dies von X“ vorzuliegen scheint und die Grammatikalisierung hin $\mathrm{zu}$ einem „diese X“ noch nicht stattgefunden hat: jnk $n w n$ ŠSyt.t ",ich bin dieser der Schaitet“" (3x, sonst nicht belegte mutmaßliche Göttin) $)^{325}, m$ jz.t $R^{c}(m) n w n$ mšr.w (4× $)^{326}$, wenn richtig mit Barguet $(1986,583)$,parmi les équipages de Rê, ceux du soir“ (Faulkner 1973/8, I, 110 liest hingegen „in the crew of Re' in these evenings“). Dazu kommt als dritter Beleg jr $n w$ n $n$ tnm.w ,was diese Kesseldämonen betrifft“ $(1 \times)^{327}$, doch ist dies eine in einer Glosse stehende verkürzte Wiederholung des zuvor im Text genannten $n w$ $n$ tnm.w spd.w $d b^{c} . w$,diese Kesseldämonen mit spitzen Fingern“ $(2 \times)^{328}$ mit komplexem Rectum. Trotz der schwachen Beleglage dürfen wir davon ausgehen, dass $n w$ mit unerweitertem Rectum den indirekten Genitiv erfordert.

In den Sargtexten viel geläufiger ist $n w$ plus Genitiv dann, wenn das Rectum komplex ist, also seinerseits Attribute beinhaltet. Auch dann wird in aller Regel der indirekte Genitiv verwendet. Ich zitiere nur eine Auswahl von Belegen: $n w n$ bw.wt ntr. $(w)$,diese Abscheue des Gottes/ der Götter“ $(7 \times)^{329}, n w n h^{c} . w(n) r m t . w$,diese menschlichen Glieder" $(6 \times)^{330}$, nw n ntr.w jmj.w šmsw Wsjr „diese Götter, die im Gefolge des Osiris sind“ $(4 \times)^{331}, n w n$ šd.w $n m w . w$,diese Grundstücke der Zwerge“ $(4 \times)^{332}, n w n$ 3h.w jmj.w j3hw „diese Totengeister, die im Sonnenlicht sind“ $(3 \times)^{333}, n w n$ fnt.w qm3.n jr.t $n b w^{c}$, diese Würmer, die das Auge des einzigen Herrn schuf " $(3 \times)^{334}, n w n t . w w^{c} b . w$ pri „diese reinen Brote, die hervorkamen“ $(3 \times)^{335}$, nw $n$ mw $w^{c} b . w$ pri.w $m w^{c}$ r.t tw n.t $n$ '.tjw „dieses reine Wasser, das aus diesem Uferland der Stadtbewohner hervorkam“

324 CT III 50c, IV 79e, VI 290i, VI 327a.

325 CT V 64e.

326 CT II 140b.

327 CT IV $304 a$.

328 CT IV $303 a$.

329 CT IV 324b, IV 3300.

330 CT II 228a.

331 CT I 54 h.

332 CT II 153a, II 161c.

333 CT IV 73f, IV 75a.

334 CT IV 76a.

335 CT III 257a.
$(2 \times)^{336}, n w n$ nhnw sd $d r$ jwi $m q 33 b$ mw.t= $f$,dieser Jüngling, der hilflos im Leib seiner Mutter liegt“ $(2 \times)^{337}, n w n$ šnw $n t j$ $r h h=s$, diese Haare, die an ihrem Hals sind“ $(1 \times)^{338}$.

Nicht selten steht im Rectum ein adjektivisches Lexem, das in dieser Konstruktion aber offenbar substantiviert ist: $n w n$ jy $\underline{\underline{d}} \underline{d} s=f$, dieser/derjenige, der von selbst gekommen ist“ ( $(1 \times)^{339}, n w n$ swnw $h r$, diese mit leidendem Gesicht“ $(2 \times)^{340}, n w$ n jmj.w-ht Hrw „diese Gefolgsleute des Horus“ (35× $)^{341}, n w$ n jmj.w 3h.t ,diese, die im Horizont sind“ $(1 \times)^{342}$, nw n jrj.w st $3 . w$, diese zum Schlachten gehörigen“ $(8 \times)^{343}, n w$ n jrj.w sjp.w „diese zum Verhör gehörigen“ $(5 \times)^{344}$. Wenn in ähnlichem Kontext das $n$ fehlt, kann man das nicht als Beleg für einen direkten Genitiv nehmen, sondern das Adjektiv könnte hier seine ursprüngliche Wortart behalten haben: $n w$ jrj rmn.wj $R^{c}$ „dies, was sich zu beiden Seiten des Re befindet“ $(3 x)^{345}$. Wohl durch die Unsicherheit, ob das Adjektiv substantiviert ist oder nicht, sind die folgenden Fälle von Variation zu erklären: $n w n$ phr $p$.t „dies, was den Himmel durchquert“ $(6 \times) \sim n w$ phr p.t $(1 \times)^{346}, n w n{ }^{c} 3$ jti $i$,dieser Große, der erobert (?)“ $(2 \times) \sim n w{ }^{c} j \underline{j} \underline{i}(1 \times)^{347}$. Der Fall, dass das Regens $n w$ durch weitere Attribute erweitert wäre, ist aus semantischen Gründen kaum denkbar und kommt nicht vor.

Die Stelle $m 33 n w n t r . w n m d w$ jmn.t nfr.t $r$ NN pn $(10 \times)^{348}$, die ein abnormes * $n w$ ntr. $w$,diese Götter“ zu enthalten scheint, hat den Übersetzern Schwierigkeiten bereitet. Ich nehme an, dass hier ntr. $w$ als Vokativ eingeschoben ist (wie in dem ähnlichen Fall CT II 218b), und zwar in die indirekte Genitivgruppe $n w n m d w$ hinein. Die korrekte Übersetzung fand bisher nur Jürgens (1995, 282f.): „Beachtet, ihr Götter, (diese) Worte des Schönen Westens zu dem verstorbenen $N$. “ aufgrund seiner Beobachtung, dass m33 in zwei Textzeugen durch Pluralstriche explizit als Imperativ Plural gekennzeichnet ist. Doch wie seine Transliteration $m 3(. w), n w<n(. i)>n t r(. w), n m d w(. w)$ zeigt, lag er noch nicht völlig richtig, sondern wollte $n w$ zu $n t r . w$ ziehen und wurde dadurch sowohl zu einer Emendation

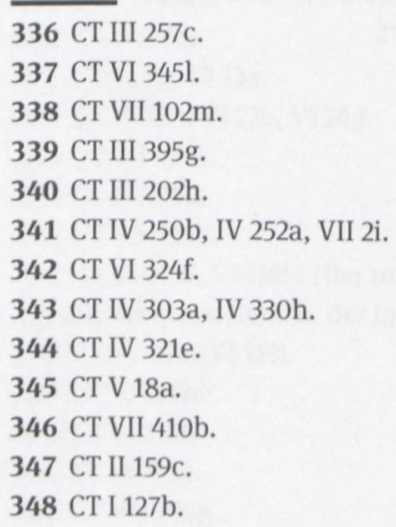


als auch zur Annahme einer ungewöhnlichen dativischen Rektion von $m 33$,sehen“ gezwungen.

Die vier zuletzt behandelten Lemmata $j b, r n, k 3$ und $n w$ sind Beispiele für eine Klasse von Substantiven, die folgende Eigenschaften hat: (1) Auf sie folgt in der Regel der indirekte Genitiv, sowohl bei einfachem wie bei erweitertem Rectum. (2) Einige sporadische Belege mit direktem Genitiv sind aber vorhanden. (3) Bei erweitertem Regens steht immer der indirekte Genitiv. (4) Mehrfach ist zu beobachten, dass der Plural dieser Substantive sich anders verhält und vorwiegend den direkten Genitiv zu sich nimmt.

In dieser Klasse befinden sich einige Körperteile, so dass man annehmen könnte, der indirekte Genitiv stünde bei inalienabler Possession. Dagegen sehe ich vier Gegenargumente: (1) Die Klasse enthält nicht nur Körperteile. (2) Die Distribution wäre gerade umgekehrt wie zu erwarten, denn im inalienablen Fall sollte die einfachere, direktere Konstruktion stehen: „a relationship which is semantically close - such as inalienable possession (...) - is likely to employ a simpler or tighter grammatical marking than one which is semantically neutral - such as alienable possession“ (Dixon 2010-12, II, 286). (3) Das unterschiedliche Verhalten von Singular- und Pluralformen spricht gegen eine solche funktionale Motivation. (4) Sprachen, die einen Unterschied zwischen alienabler und inalienabler Possession machen, tun dies auch und insbesondere bei pronominaler Possession, was im Älteren Ägyptisch nicht der Fall ist. Ich vermute eher eine gemeinsame formale Eigenschaft der zu dieser Klasse gehörigen Substantive und sehe höchstens einen indirekten Zusammenhang mit der Körperteilsemantik: In dieser Klasse scheinen sich insbesondere sehr kurze Substantive zu befinden. Kurze Lautkörper sind aber typisch für besonders häufige und elementare Ausdrücke, darunter eben auch Bezeichnungen von Körperteilen im Singular.

\section{Fazit}

Ich habe zwei Klassen von Substantiven vorgestellt, nämlich eine, die nach sich regelmäßig den direkten Genitiv erfordert, und eine, die regelmäßig den indirekten Genitiv erfordert. Im einzelnen konnten noch weitere Regeln aufgestellt werden. Mit diesen beiden Klassen ist das Thema aber noch nicht erledigt: Es gibt auch Substantive, die sowohl mit direktem als auch mit indirektem Genitiv häufig stehen. Diese beabsichtige ich in einem künftigen Aufsatz zu behandeln. Auch sollten die Faktoren noch identifiziert werden, welche die Zugehörigkeit eines Substantivs zu einer der Klassen bestimmen.

Diese Regeln können auch beim Übersetzen helfen, denn hier bringt ja jede neue grammatische Regel ein Stückchen mehr Sicherheit. So bedeutet beispielsweise $\underline{d i}=k$ w3.t $n \underline{d}$ 3.t $r$ p.t (CT I 264h) nicht ,grant the crane's way to the sky" (Faulkner 1973/8, I, 57) - schon wegen der fehlenden Kongruenz -, sondern „tu montres le chemin à la grue vers le ciel“ (Barguet 1986, 200). Auch jr tm wnn z3 $n$ z (CT VI 333i) muss mit Dativ gelesen werden. Diesmal liegt Faulkner (1973/8, II, 264) richtig: „If there be not a son for a man“, falsch hingegen Barguet $(1986,608)$ „s'il n'y a pas de fils d'un homme“ und Carrier $(2004,1598)$, der explizit $s_{3} n(y) s$ transkribiert. In sšm k3.w n nb.w hr.t (CT V 363d) muss ebenfalls ein Dativ vorliegen, und so liegt Barguet $(1986,78)$ wieder richtig: „celui qui conduit les subsistances aux Maîtres des biens" im Gegensatz zu Faulkners (1973/8, II, 95) „one whom the spirits of the Lords of Plenty guide“. Eine Uneinigkeit bezüglich der Wortsegmentierung liegt vor in $j b n g 3$ ht.t (CT VI 47b). Hier ist kaum mit Faulkner (1973/8, II, 126) zu lesen ,the heart of him whose neck was broken ( $\left.n g^{3}\right)$ “ (das ghost-word $n g$ 3) ist auch in van der Molen 2000, 252 eingegangen), sondern „le cœur de celui dont la gorge est resserrée $(g$ ')“ (Barguet 1986, 316). Dies ist keineswegs als Kritik an den bisherigen Übersetzern gemeint, sondern soll nur illustrieren, wie die Philologen unweigerlich ins Schwimmen kommen, solange die Grammatik nicht hinreichend geklärt ist.

\section{Bibliographie}

Altenmüller, H. 1966, „'Messersee', 'gewundener Wasserlauf' und 'Flammensee'“, ZÄS 92, 86-95.

Backes, B. 2005, Das altägyptische »Zweiwegebuch «. Studien zu den Sargtext-Sprüchen 1029-1130, Wiesbaden.

Bakir, 'Abd el-M. 1966, „Varia grammatica“, JEA 52, 34-38.

Barguet, P. 1986, Les textes des sarcophages égyptiens du moyen empire, Paris.

Barta, W. 1995, „Die Präpositionalnisbe $n j$ in einfacher und umgekehrter Bedeutung“, in Kessler, D. \& Schulz, R. (Hrsgg.), Gedenkschrift für Winfried Barta, Frankfurt, 29-35.

Borghouts, J. F. 2010, Egyptian. An introduction to the writing and language of the Middle Kingdom, 2 Bde., Leuven.

Brose, M. 2014, Grammatik der dokumentarischen Texte des Mittleren Reiches, Hamburg.

de Buck, A. 1949, „The Earliest Version of the Book of the Dead“, JEA 35, 87-98.

de Buck, A. \& Gardiner, A. H. 1935 ff., The Egyptian Coffin Texts, 8 Bde., Chicago.

Callender, J. B. 1975, Middle Egyptian, Malibu.

Carrier, C. 2004, Textes des sarcophages du moyen empire égyptien, 3 Bde., Monaco. 
Černý, J. \& Groll, S. I. 1984, A Late Egyptian grammar, 3rd ed., Rome. Depuydt, L. 1999, „Egyptian 'split' genitives and related phenomena: Exotic debris from conflicting forces“, Le Muséon 112, 273-299.

Dixon, R. M. W. 2010-12, Basic linguistic theory, 3 Bde., Oxford.

Doret, E. 1989, „Sur une caractéristique grammaticale de quelques sarcophages d'el-Bersheh", BSEG 13, 45-50.

Edel, E. 1955/64, Altägyptische Grammatik, Roma.

Egedi, B. 2010, „Possessive constructions in Egyptian and Coptic“, ZÄS 137, 1-12.

Erman, A. 1889, Die Sprache des Papyrus Westcar, Göttingen.

- 1928, Ägyptische Grammatik, 4. Aufl., Berlin.

Faulkner, R. 0. 1969, The Ancient Egyptian Pyramid Texts, Supplement of hieroglyphic texts, Oxford.

- 1973/8, The Ancient Egyptian Coffin Texts, 3 Bde., Warminster.

Gardiner, A. H. 1957, Egyptian grammar, 3. Aufl., London.

Grandet, P. \& Mathieu, B. 1990, Cours d'égyptien hiéroglyphique, vol. I, Paris.

Gunn, B. 1949, „A special use of the śdm.f and śdm.n.f forms“, JEA 35, 21-24.

Hannig, R. 2006, Ägyptisches Wörterbuch II: Mittleres Reich und Zweite Zwischenzeit, 2 Bde., Mainz

Jansen-Winkeln, K. 1996, Spätmittelägyptische Grammatik der Texte der 3. Zwischenzeit, Wiesbaden.

- 2000, „Bemerkungen zum «Genetiv» im Ägyptischen“, ZÄS 127, 27-37.

Jenni, H. 2010, Lehrbuch der klassisch-ägyptischen Sprache, Basel. Junge, F. 1999, Einführung in die Grammatik des Neuägyptischen, 2. Aufl., Wiesbaden.

Junker, H. 1938, Gîza III, Wien.

Jürgens, P. 1995, Grundlinien einer Überlieferungsgeschichte der altägyptischen Sargtexte, Wiesbaden.

Kammerzell, F. 2000, „Egyptian possessive constructions: a diachronic typological perspective“, Sprachtypologie und Universalienforschung 53, 97-108.

Krauss, R. 1997, Astronomische Konzepte und Jenseitsvorstellungen in den Pyramidentexten, Wiesbaden.
Lefebvre, G. 1955, Grammaire de l'égyptien classique, Le Caire.

Luft, U. 1978, Beiträge zur Historisierung der Götterwelt und der Mythenschreibung, Budapest.

Molen, R. van der 2000, A hieroglyphic dictionary of Egyptian coffin texts, Leiden.

Sander-Hansen, C. E. 1936, „Zum Gebrauch des direkten und indirekten Genitivs in der ältesten ägyptischen Sprache“, Acta Orientalia (Havniæ) 14, 26-54.

- 1963, Ägyptische Grammatik, Wiesbaden.

Scheele-Schweitzer, K. 2014, Die Personennamen des Alten Reiches, Wiesbaden.

Schenkel, W. 1963, „Direkter und indirekter «Genitiv» “, ZÄS 88, 58-66.

- 2011, „Merkmalloses versus pluralisches/distributives/intensives Partizip“, ZÄS 138, 63-78.

- 2012, Tübinger Einführung in die klassisch-ägyptische Sprache und Schrift [grüne Auflage], Tübingen.

Schweitzer, S. 2005, Schrift und Sprache der 4. Dynastie, Wiesbaden.

Sethe, K. 1935 ff., Übersetzung und Kommentar zu den altägyptischen Pyramidentexten, 6 Bde., Hamburg.

Shisha-Halevy, A. 2007, „Determination-signalling environment in Old and Middle Egyptian: Work-notes and reflections", in Bar, T. \& Cohen, E. (Hrsgg.), Studies in Semitic and general linguistics in honor of Gideon Goldenberg, Münster, 223-254.

Sperveslage, G. 2010, „Das Kongruenzverhalten von Adjektiven im Altägyptischen“, Lingua Aegyptia 18, 217-251.

Spiess, H. 1997, „Der direkte Genitiv“, Discussions in Egyptology 37, 65-72.

el Toukhy, A. 1993, „Nw in den Sargtexten“, GM 132, 33-36.

Vernus, P. 1994, „Un emploi particulier du démonstratif $n w$ dans les textes des sarcophages“, in Berger, C. et al. (Hrsgg.), Hommages à Jean Leclant, Bd. 4, BdÉ 106/4, Le Caire, 411-415.

Warburton, D. A. 2015, „The syntax of possession“, in Nyord, R. \& Ryholt, K. (Hrsgg.), Lotus and Laurel (Fs Frandsen), Copenhagen, 431-446.

Westendorf, W. 1962, Grammatik der medizinischen Texte, Berlin. 